\title{
Population and Mortality Predictions of the Florida Manatee - Ten Year Watercraft Mortality
}

Dylan Lehigh

University of South Florida

\author{
Advisors: \\ Arcadii Grinshpan, Mathematics and Statistics \\ Jeffrey A. Hostetler, Research Scientist, Marine Mammal Program, Florida Fish and Wildlife \\ Conservation Commission, Fish and Wildlife Research Institute
}

Problem Suggested By: Jeffrey A. Hostetler, Dylan Lehigh, and Roy Yanong (University of Florida, Tropical Aquaculture Laboratory)

Follow this and additional works at: https://digitalcommons.usf.edu/ujmm

Part of the Mathematics Commons

UJMM is an open access journal, free to authors and readers, and relies on your support:

Donate Now

\section{Recommended Citation}

Lehigh, Dylan (2019) "Population and Mortality Predictions of the Florida Manatee - Ten Year Watercraft Mortality," Undergraduate Journal of Mathematical Modeling: One + Two: Vol. 9: Iss. 2, Article 5.

DOI: https://doi.org/10.5038/2326-3652.9.2.4904

Available at: https://digitalcommons.usf.edu/ujmm/vol9/iss2/5 


\title{
Population and Mortality Predictions of the Florida Manatee - Ten Year Watercraft Mortality
}

\begin{abstract}
The West Indian Manatee is currently listed as a threatened species by the U.S Fish and Wildlife service. A threatened designation means population levels still are at risk of becoming endangered again in the foreseeable future. This project seeks to forecast how many manatees would perish from watercraft strikes (the greatest threat to their survival) based upon the most current method utilized for projecting manatee population growth.

The latest version, known as the Core Biological Model (version 6) or CBMv6, was employed along with the most current manatee population data, which was provided by Dr. Jeffrey A. Hostetler. Dr. Hostetler is the predominate research scientist for the Florida Manatee population. The most current Florida Manatee total population stands at: 6,352 .

The results sought are the total number of watercraft deaths, expected for the next 10 consecutive years. The calculation will require, from the current population, predicting the population growth factors for each of the four regions (ATL, USJ, NW, and SW), with segregation by sex, age, and reproductive status.
\end{abstract}

\section{Keywords}

Florida manatee population, mortality prediction, watercraft strike, Core Biological Model, modeling matrix, survival rate, reproductive rate

\section{Creative Commons License}

(c) (i) (8)

This work is licensed under a Creative Commons Attribution-Noncommercial-Share Alike 4.0 License. 


\section{PROBLEM STATEMENT}

The goal of this project is to predict the total amount of West Indian manatees killed by watercraft strikes over the course of the next 10 years.

\section{MOTIVATION}

The West Indian Manatee, also known as the Florida Manatee (Trichechus manatus Latirostris), is found throughout the coastal waters of Florida, and its rivers. On March 30 ${ }^{\text {th }}, 2017$ the U.S Fish and Wildlife Service reclassified the once Endangered animal to Threatened (www.fws.gov). Under these terms Threatened means that the population is likely to become Endangered again in the foreseeable future without safeguards. Given their Threatened status the species still has certain protections afforded by the Endangered Species Act (under the Marine Mammal Protection Act). They include Manatee protection areas, and population monitoring.

The four regions are Atlantic, Upper St. John's River, Northwest, and Southwest. Based upon the most current population numbers provided by Dr. Hostetler (2017) the population of manatees consists of:

\begin{tabular}{|l|r|}
\hline starting population (survey) ALT: & 3221 \\
\hline starting population (survey) USJ: & 340 \\
\hline starting population (survey) NW: & 610 \\
\hline starting population (survey) SW: & 2181 \\
\hline & \\
\hline Total population: & 6352 \\
\hline
\end{tabular}

That being said, Watercraft strikes are the most prevalent cause of death for manatees. In order to illustrate the future impact of watercraft strikes on the manatee population the most current version of the Common Biological Model (CBMv6) is applied. The purpose of this paper is to calculate the expected total watercraft deaths of Florida manatees for the next 10 years. Due to differing survival rates within the four regions, a separate calculation will be required for each region (ATL, USJ, NW, SW), which will include predicting future population growth. 


\section{MATHEMATICAL DESCRIPTION AND SOLUTION APPROACH}

The latest Common Biological Model (CBMv6) seeking to forecast the amount of manatees that died per year by watercraft strikes for the next 10 years is applied.

The CBMv6 is a predictive modeling matrix used to calculate the future population by stages. These states are assigned from "Status and Threats Analysis for the Florida Manatee", 2016 (Runge, Sanders-Reed and Langtimm), below Figure one from Runge.

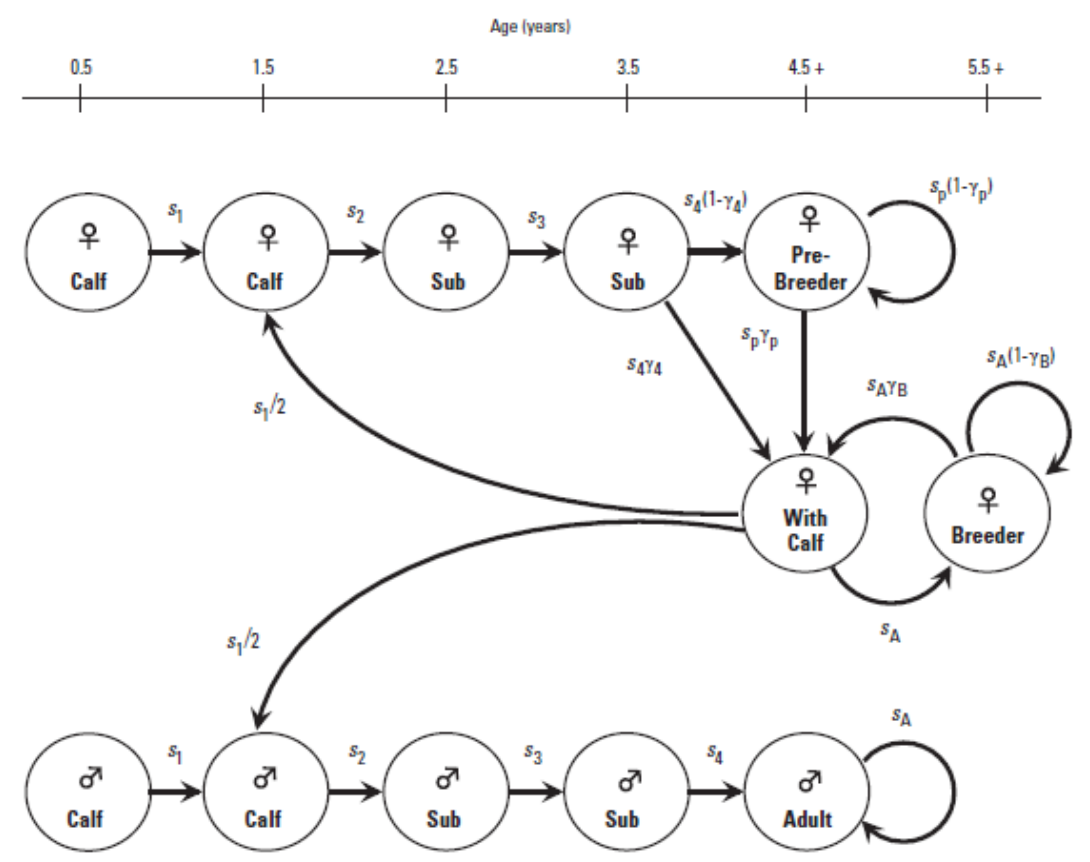

Figure 1. Life-history stages and transitions for the manatee population model. Sex-and stage-specific survival (s) and reproductive $(\gamma)$ rates govern the transition between stages. Females $(\%)$ and males $\left(\sigma^{7}\right)$ are separately accounted for in the model. Calves enter the population model as separate entities at 1.5 years; until that time, they are counted as part of the female-with-calf pair. (The 0.5-year stage is shown for completeness but is not tracked separately in the model) 1

The matrix applied for predicting population mortality, growth, and reproduction (Figure 2):

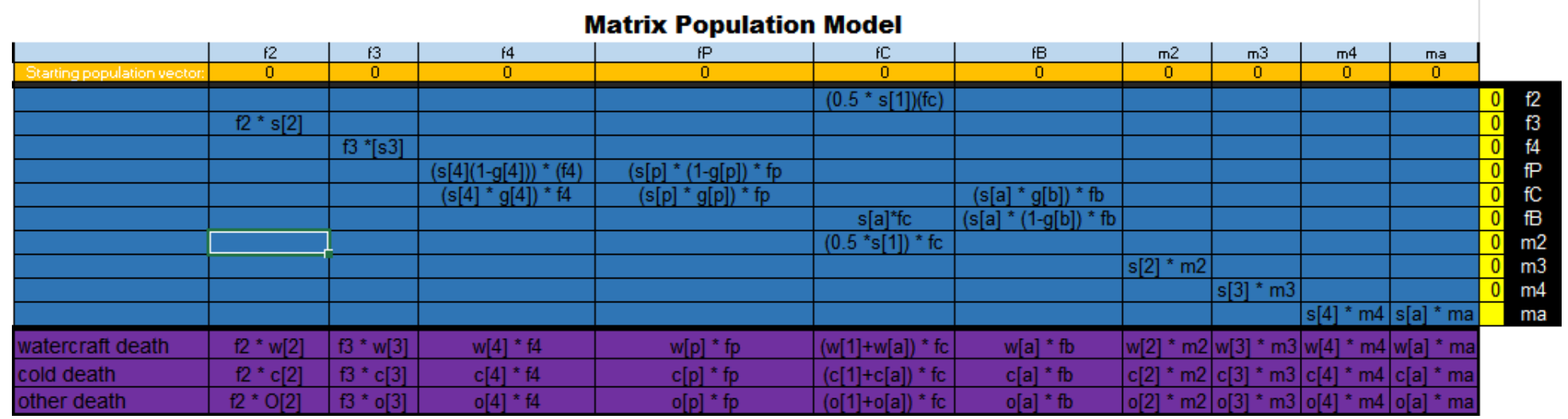

Figure 2-CBMv6 (Common Biological Model) 
The model utilizes stages assigned (see figure $3^{1}$ below) by sex, age, and reproductive status (i.e. $\mathbf{f 1}=$ female calf $0.5-1.5$ years of age, $\mathbf{f} 2=$ female calf $1.5-2.5$ years of age, and so on (see figure 3)). Each region must be independently calculated by each stage (f2, f3, fA, excreta) due to differing survival (see figure 3) and reproductive rates within the four regions, which results in the watercraft mortality projection (figure 4).

\begin{tabular}{|c|c|}
\hline 12 & s[2] female ( $1.5-2.5$ years) \\
\hline f3 & $s[3]$ female ( $2.5-3.5$ years] \\
\hline$f 4$ & s[4] female ( $3.5-4.5$ years] \\
\hline $\mathrm{fP}$ & $s[\mathrm{P}] \mathrm{Pre}$-breeder female ( +4.5 years] \\
\hline $\mathrm{fC}$ & $s[\mathrm{~A}]$ female Adult with calf ( +4.5 years) \\
\hline $\mathrm{fB}$ & s[A] female Adult ( +4.5 years] \\
\hline $\mathrm{m} 2$ & $s[2]$ male $(1.5-2.5$ years $]$ \\
\hline $\mathrm{m} 3$ & $s[3]$ male $(2.5-3.5$ years $]$ \\
\hline $\mathrm{m} 4$ & $s[4]$ male $(3.5-4.5$ years $)$ \\
\hline $\mathrm{ma}$ & $s[\mathrm{~A}]$ male adult \\
\hline
\end{tabular}

Figure 3 - Stages

\begin{tabular}{|l|r|r|r|r|}
\hline $\begin{array}{l}\text { Survival } \\
\text { rate }\end{array}$ & ATL & USJ & NW & \multicolumn{1}{c|}{ SW } \\
\hline $\mathrm{S}[1]$ & 0.8356 & 0.8665 & 0.9261 & 0.94 \\
\hline $\mathrm{S}[2]$ & 0.8726 & 0.8538 & 0.9428 & 0.9343 \\
\hline $\mathrm{S}[3]$ & 0.9729 & 0.979 & 0.978 & 0.9759 \\
\hline $\mathrm{S}[4]$ & 0.9729 & 0.979 & 0.978 & 0.9759 \\
\hline $\mathrm{S}[\mathrm{P}]$ & 0.9729 & 0.979 & 0.978 & 0.9759 \\
\hline $\mathrm{S}[\mathrm{A}]$ & 0.9729 & 0.979 & 0.978 & 0.9759 \\
\hline
\end{tabular}

Figure 4 Survival rates by region

The model predicts the total population for a region, broken down into ten stages (Figure 3 and Figure 1) of the manatee life cycle based on age, sex, and reproductive status. This is done by multiplying the matrix by a starting population or population vector.

This $\mathrm{S}[\mathrm{x}]$ represents the $S$ urvival rates of the manatees in a specific region, and $\mathrm{g}[\mathrm{x}]$ represents the reproductive rates. Each row of the matrix calculates the predicted population and mortalities.

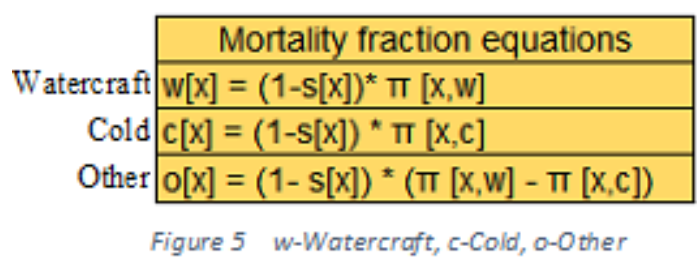

Reproductive rates

$\mathrm{g}[4]$

$\mathrm{g}[\mathrm{B}]$

$\mathrm{g}[\mathrm{P}]$

Figure 7 Reproductive rates

\begin{tabular}{|c|c|c|}
\cline { 2 - 3 } \multicolumn{1}{c|}{} & $\pi[\mathrm{x}, \mathrm{W}]$ & $\pi[\mathrm{x}, \mathrm{c}]$ \\
\hline$[1]$ & 0.6008 & 0.1661 \\
\hline$[2]$ & 0.6008 & 0.1661 \\
\hline$[3]$ & 0.7927 & 0.0454 \\
\hline$[4]$ & 0.7927 & 0.0454 \\
\hline$[\mathrm{p}]$ & 0.7927 & 0.0454 \\
\hline$[\mathrm{a}]$ & 0.7927 & 0.0454 \\
\hline
\end{tabular}

Figure 6 Mortality fractions for watercraft mortalities and cold snap mortalities

\footnotetext{
${ }^{1}$ Stages are assigned by (Runge, Sanders-Reed and Langtimm)
} 


\section{DISCUSSION}

As anticipated, the model predicts a rising population, which naturally increases mortality numbers as the population grows. In ten years' time the total number of manatee mortalities as a result of watercraft is predicted to reach 2,395 in total across the four regions of Florida.

For perspective, 2,395 manatee deaths as a result of watercraft in ten years is more than one-third of the entire present population (6,352 total manatees). However, the model also predicts a manatee population increasing in the next 10 years to 9,529 .

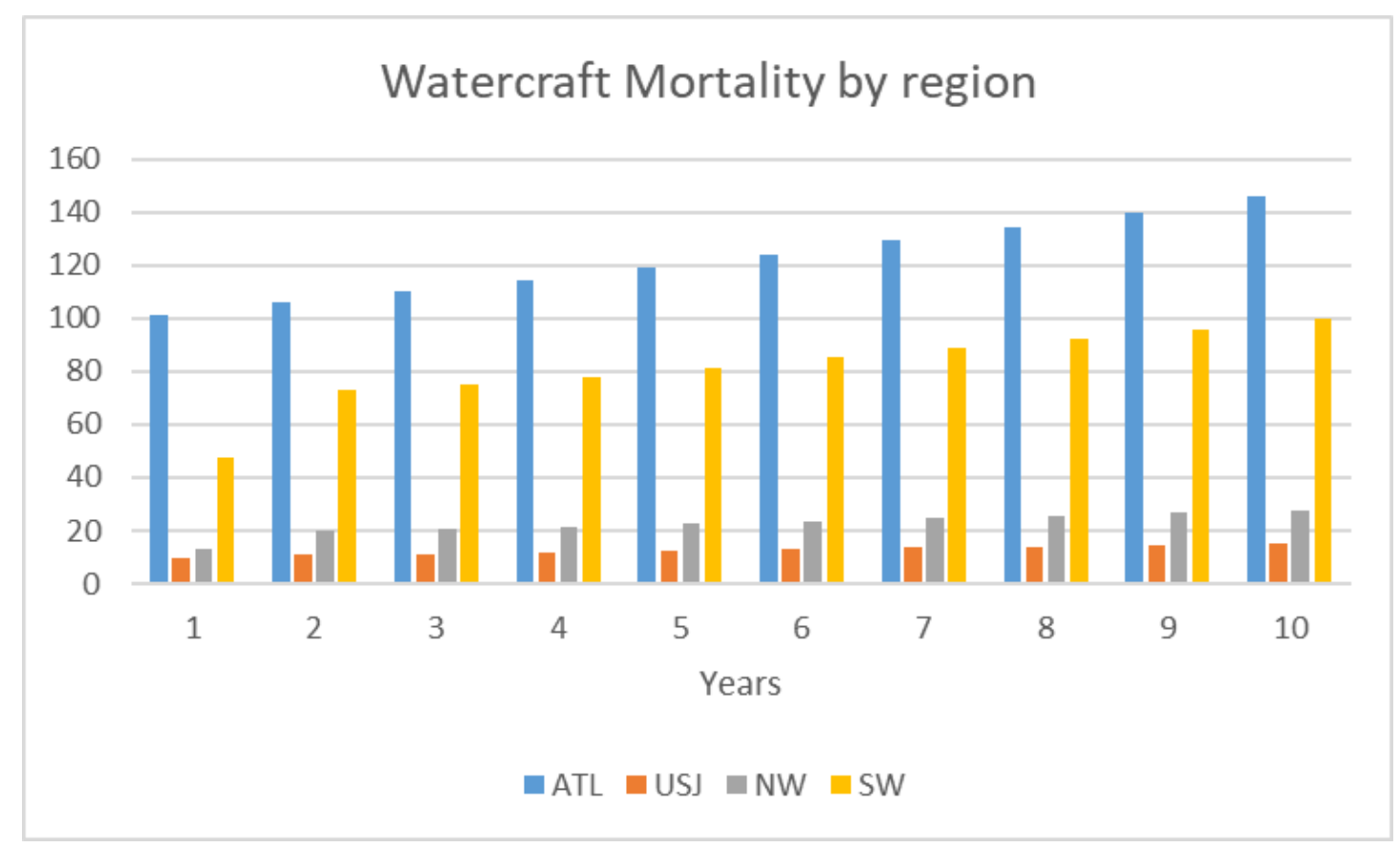

By comparison, the model predicts approximately 356 deaths from cold weather conditions and 672 deaths related to other causes in the next 10 years, which combined is less than half than the predicted watercraft deaths. Thus, at present the greatest single mortality risk for manatees are watercraft deaths. 


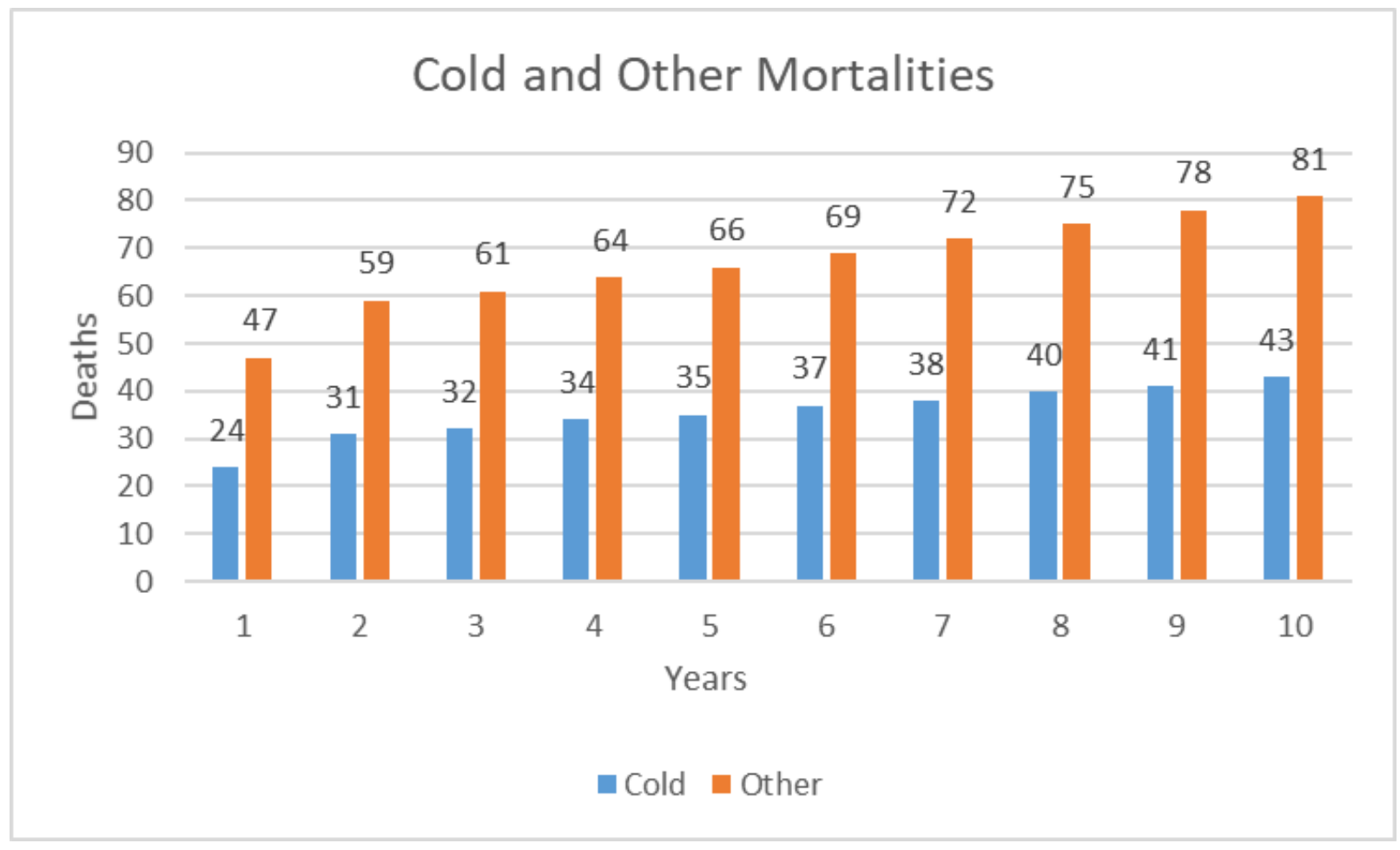




\section{CONCLUSIONS AND RECOMMENDATIONS}

By comparison the single greatest threat to the Florida manatee is watercraft, which is more than double all other mortality causes. Thus the majority of deaths are a consequence of human behavior. While most Floridians are aware of the threat to manatees, the magnitude can be put into better perspective when projecting over a ten-year period. Perhaps human behavior would be impacted, to the benefit of manatees, if it were common knowledge that the leading cause of manatee deaths is associated with watercraft.

Based upon the aforementioned calculation results, perhaps greater care and caution would be taken in the manatee habitat if there were awareness that over the next ten years more than one third of the present day population is predicted to die as a result of watercraft. Perhaps a campaign utilizing a ten-year projection would help public perception of these grim realities. 


\section{NOMENCLATURE}

\begin{tabular}{|c|c|c|}
\hline ATL & - & Atlantic Management Unit \\
\hline s & - & Survival rate \\
\hline$\pi$ & - & [also pi] fraction of mortality \\
\hline $\mathrm{g}$ & - & Reproductive rates \\
\hline $\mathrm{x}$ & - & $=$ stage $x$ (i.e. $\mathrm{s}[1], \mathrm{s}[\mathrm{P}], \mathrm{s}[\mathrm{A}] \ldots$ ) \\
\hline ESA & - & Endangered Species Act \\
\hline $\mathrm{F} 2$ & - & Female Calf Age 1.5-2.5 \\
\hline F3 & - & Female sub Adult Age 2.5-3.5 \\
\hline $\mathrm{F} 4$ & - & Female Sub Adult Age 3.5-4.5 \\
\hline FP & - & Female Prebreeder Age $4.5-5.5$ \\
\hline FB & - & Female with Calf Age $4.5-5.5$ \\
\hline M2 & - & Male Calf Age 1.5-2.5 \\
\hline M3 & - & Male Sub Adult Age 2.5-3.5 \\
\hline M4 & - & Male Sub Adult Age 3.5-4.5 \\
\hline MA & - & Male Adult Age 4.5-5.5 \\
\hline Other & - & Death by any other means than water craft and cold snap \\
\hline
\end{tabular}




\section{BIBLIOGRAPHY}

Runge, Michael C., et al. "Status and threats analysis for the Florida manatee (Trichechus manatus latirostris), 2016." US Gelogical Survey (2017).

www.fws.gov. 30 March 2017. electronic. 10 december 2018.

www.fws.gov. 5 October 2018. electronic. 10 December 2018. 


\title{
Appendix
}

\author{
Table of Contents
}

\begin{abstract}
ATL
$\underline{\text { USJ }}$

NW

$\underline{\mathrm{SW}}$
\end{abstract}

In consideration for how small the print would be I have limited the prediction model to one per page for ease of view. 


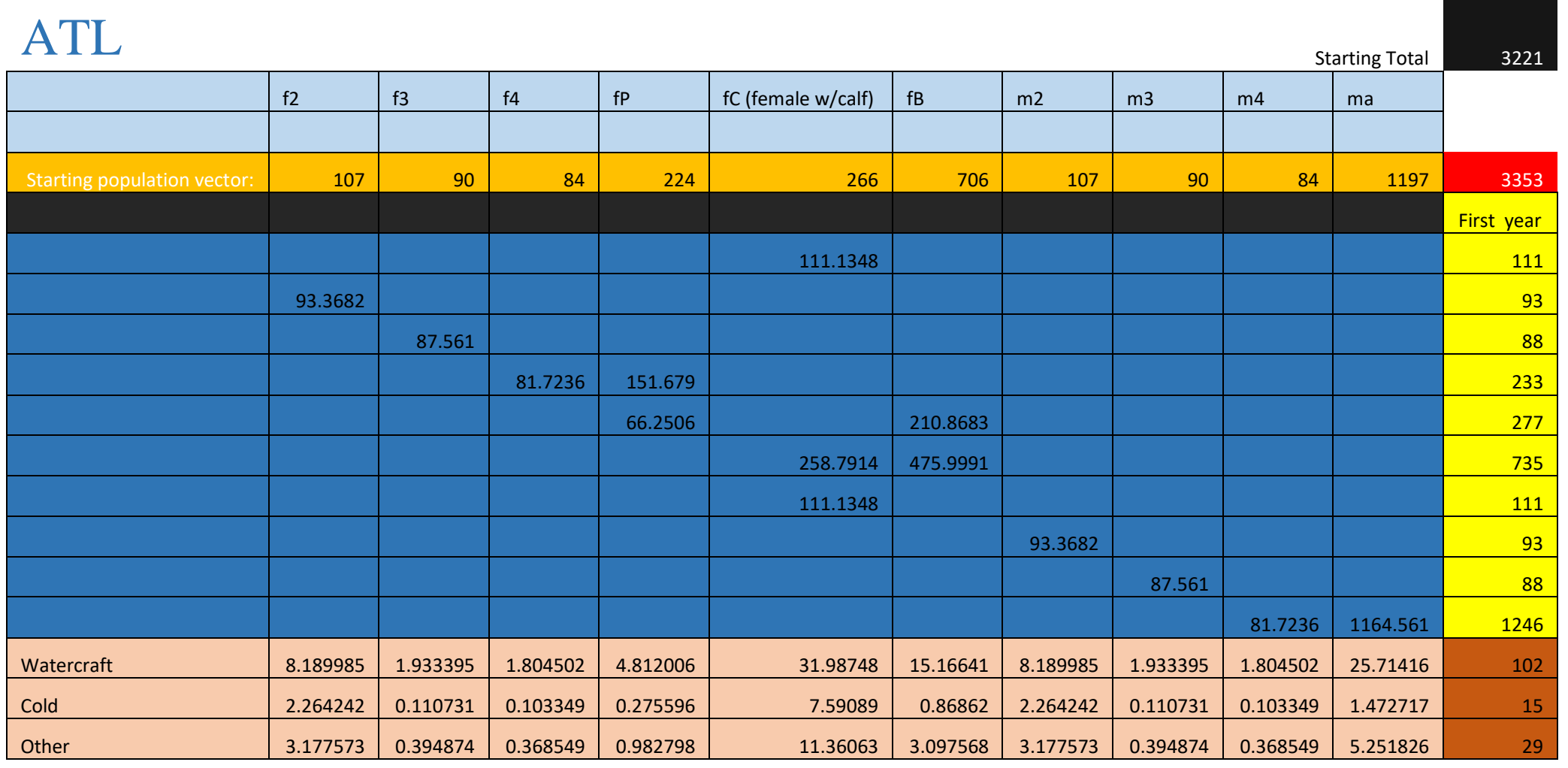




\begin{tabular}{|c|c|c|c|c|c|c|c|c|c|c|c|}
\hline & $\mathrm{f} 2$ & $\mathrm{f3}$ & $\mathrm{f} 4$ & fP & fC (female w/calf) & $\mathrm{fB}$ & $\mathrm{m} 2$ & m3 & $\mathrm{m} 4$ & ma & \\
\hline \multirow[t]{12}{*}{ Starting population vector: } & 111 & 93 & 88 & 233 & 277 & 735 & 111 & 93 & 88 & 1246 & 3490 \\
\hline & & & & & & & & & & & second year \\
\hline & & & & & 115.7803 & & & & & & 116 \\
\hline & 96.97623 & & & & & & & & & & 97 \\
\hline & & 90.83792 & & & & & & & & & 91 \\
\hline & & & 85.1881 & 158.0459 & & & & & & & 243 \\
\hline & & & & 69.03153 & & 219.4674 & & & & & 288 \\
\hline & & & & & 269.609 & 495.4102 & & & & & 765 \\
\hline & & & & & 115.7803 & & & & & & 116 \\
\hline & & & & & & & 96.97623 & & & & 97 \\
\hline & & & & & & & & 90.83792 & & & 91 \\
\hline & & & & & & & & & 85.1881 & 1212.511 & 1298 \\
\hline Watercraft & 8.506471 & 2.005752 & 1.881 & 5.013994 & 33.32457 & 15.78489 & 8.506471 & 2.005752 & 1.881 & 26.7729 & 106 \\
\hline Cold & 2.351739 & 0.114875 & 0.10773 & 0.287165 & 7.908192 & 0.904042 & 2.351739 & 0.114875 & 0.10773 & 1.533354 & 16 \\
\hline Other & 3.300363 & 0.409652 & 0.384173 & 1.024052 & 11.83551 & 3.223886 & 3.300363 & 0.409652 & 0.384173 & 5.468063 & 30 \\
\hline
\end{tabular}




\begin{tabular}{|c|c|c|c|c|c|c|c|c|c|c|c|}
\hline & $\mathrm{f} 2$ & $\mathrm{f3}$ & $\mathrm{f} 4$ & $\mathrm{fP}$ & $\mathrm{fC}$ (female w/calf) & $\mathrm{fB}$ & $\mathrm{m} 2$ & $\mathrm{~m} 3$ & $\mathrm{~m} 4$ & ma & \\
\hline \multirow[t]{12}{*}{ Starting population vector: } & 116 & 97 & 91 & 243 & 288 & 765 & 116 & 97 & 91 & 1298 & 3633 \\
\hline & & & & & & & & & & & Third year \\
\hline & & & & & 120.5349 & & & & & & 121 \\
\hline & 101.0299 & & & & & & & & & & 101 \\
\hline & & 94.34817 & & & & & & & & & 94 \\
\hline & & & 88.37621 & 164.7031 & & & & & & & 253 \\
\hline & & & & 71.93927 & & 228.4962 & & & & & 300 \\
\hline & & & & & 280.6807 & 515.791 & & & & & 796 \\
\hline & & & & & 120.5349 & & & & & & 121 \\
\hline & & & & & & & 101.0299 & & & & 101 \\
\hline & & & & & & & & 94.34817 & & & 94 \\
\hline & & & & & & & & & 88.37621 & 1262.531 & 1351 \\
\hline Watercraft & 8.862044 & 2.08326 & 1.951396 & 5.225193 & 34.69307 & 16.43427 & 8.862044 & 2.08326 & 1.951396 & 27.87738 & 110 \\
\hline Cold & 2.450043 & 0.119314 & 0.111762 & 0.29926 & 8.232947 & 0.941234 & 2.450043 & 0.119314 & 0.111762 & 1.596611 & 16 \\
\hline Other & 3.43832 & 0.425482 & 0.39855 & 1.067187 & 12.32154 & 3.356514 & 3.43832 & 0.425482 & 0.39855 & 5.69364 & 31 \\
\hline
\end{tabular}




\begin{tabular}{|c|c|c|c|c|c|c|c|c|c|c|c|}
\hline & $\mathrm{f} 2$ & $\mathrm{f3}$ & $\mathrm{f} 4$ & fP & fC (female w/calf) & $f B$ & $\mathrm{~m} 2$ & m3 & m4 & $\mathrm{ma}$ & \\
\hline \multirow[t]{12}{*}{ Starting population vector: } & 121 & 101 & 94 & 253 & 300 & 796 & 121 & 101 & 94 & 1351 & 3782 \\
\hline & & & & & & & & & & & Forth year \\
\hline & & & & & 125.5219 & & & & & & 126 \\
\hline & 105.1787 & & & & & & & & & & 105 \\
\hline & & 98.29196 & & & & & & & & & 98 \\
\hline & & & 91.79134 & 171.3697 & & & & & & & 263 \\
\hline & & & & 74.85113 & & 237.8904 & & & & & 313 \\
\hline & & & & & 292.2936 & 536.9969 & & & & & 829 \\
\hline & & & & & 125.5219 & & & & & & 126 \\
\hline & & & & & & & 105.1787 & & & & 105 \\
\hline & & & & & & & & 98.29196 & & & 98 \\
\hline & & & & & & & & & 91.79134 & 1314.298 & 1406 \\
\hline Watercraft & 9.225971 & 2.170341 & 2.026803 & 5.436692 & 36.12847 & 17.10994 & 9.225971 & 2.170341 & 2.026803 & 29.02042 & 115 \\
\hline Cold & 2.550655 & 0.124301 & 0.11608 & 0.311374 & 8.57358 & 0.979931 & 2.550655 & 0.124301 & 0.11608 & 1.662075 & 17 \\
\hline Other & 3.579517 & 0.443268 & 0.413952 & 1.110383 & 12.83134 & 3.494511 & 3.579517 & 0.443268 & 0.413952 & 5.927092 & 32 \\
\hline
\end{tabular}




\begin{tabular}{|c|c|c|c|c|c|c|c|c|c|c|c|}
\hline & $\mathrm{f} 2$ & $\mathrm{f3}$ & $\mathrm{f} 4$ & $\mathrm{fP}$ & fC (female w/calf) & $\mathrm{fB}$ & $\mathrm{m} 2$ & m3 & $\mathrm{m} 4$ & $\mathrm{ma}$ & \\
\hline \multirow{12}{*}{ Starting population vector: } & 126 & 105 & 98 & 263 & 313 & 829 & 126 & 105 & 98 & 1406 & 3937 \\
\hline & & & & & & & & & & & Fifth Year \\
\hline & & & & & 130.6634 & & & & & & 131 \\
\hline & 109.5304 & & & & & & & & & & 110 \\
\hline & & 102.3284 & & & & & & & & & 102 \\
\hline & & & 95.62824 & 178.1964 & & & & & & & 274 \\
\hline & & & & 77.83293 & & 247.6927 & & & & & 326 \\
\hline & & & & & 304.2662 & 559.124 & & & & & 863 \\
\hline & & & & & 130.6634 & & & & & & 131 \\
\hline & & & & & & & 109.5304 & & & & 110 \\
\hline & & & & & & & & 102.3284 & & & 102 \\
\hline & & & & & & & & & 95.62824 & 1367.984 & 1464 \\
\hline Watercraft & 9.607689 & 2.259467 & 2.111525 & 5.65327 & 37.60832 & 17.81496 & 9.607689 & 2.259467 & 2.111525 & 30.20584 & 119 \\
\hline Cold & 2.656187 & 0.129406 & 0.120933 & 0.323778 & 8.924761 & 1.020309 & 2.656187 & 0.129406 & 0.120933 & 1.729968 & 18 \\
\hline Other & 3.727617 & 0.461471 & 0.431255 & 1.154616 & 13.35692 & 3.638504 & 3.727617 & 0.461471 & 0.431255 & 6.169201 & 34 \\
\hline
\end{tabular}




\begin{tabular}{|c|c|c|c|c|c|c|c|c|c|c|c|}
\hline & $\mathrm{f} 2$ & $\mathrm{f3}$ & $\mathrm{f} 4$ & $\mathrm{fP}$ & fC (female w/calf) & $\mathrm{fB}$ & $\mathrm{m} 2$ & m3 & m4 & $\mathrm{ma}$ & \\
\hline \multirow[t]{12}{*}{ Starting population vector: } & 131 & 110 & 102 & 274 & 326 & 863 & 131 & 110 & 102 & 1464 & 4098 \\
\hline & & & & & & & & & & & Sixth Year \\
\hline & & & & & 136.0046 & & & & & & 136 \\
\hline & 114.0169 & & & & & & & & & & 114 \\
\hline & & 106.5622 & & & & & & & & & 107 \\
\hline & & & 99.55529 & 185.4172 & & & & & & & 285 \\
\hline & & & & 80.98683 & & 257.8777 & & & & & 339 \\
\hline & & & & & 316.7039 & 582.1147 & & & & & 899 \\
\hline & & & & & 136.0046 & & & & & & 136 \\
\hline & & & & & & & 114.0169 & & & & 114 \\
\hline & & & & & & & & 106.5622 & & & 107 \\
\hline & & & & & & & & & 99.55529 & 1423.948 & 1524 \\
\hline Watercraft & 10.00123 & 2.352951 & 2.198236 & 5.882348 & 39.14566 & 18.5475 & 10.00123 & 2.352951 & 2.198236 & 31.44157 & 124 \\
\hline Cold & 2.764987 & 0.13476 & 0.125899 & 0.336897 & 9.289585 & 1.062264 & 2.764987 & 0.13476 & 0.125899 & 1.800741 & 19 \\
\hline Other & 3.880303 & 0.480564 & 0.448965 & 1.201403 & 13.90292 & 3.788116 & 3.880303 & 0.480564 & 0.448965 & 6.421584 & 35 \\
\hline
\end{tabular}




\begin{tabular}{|c|c|c|c|c|c|c|c|c|c|c|c|}
\hline & $\mathrm{f} 2$ & $\mathrm{f3}$ & $\mathrm{f} 4$ & $\mathrm{fP}$ & fC (female w/calf) & $f B$ & $\mathrm{~m} 2$ & m3 & $\mathrm{m} 4$ & ma & \\
\hline \multirow[t]{12}{*}{ Starting population vector: } & 136 & 114 & 107 & 285 & 339 & 899 & 136 & 114 & 107 & 1524 & 4266 \\
\hline & & & & & & & & & & & Seventh year \\
\hline & & & & & 141.5776 & & & & & & 142 \\
\hline & 118.6776 & & & & & & & & & & 119 \\
\hline & & 110.927 & & & & & & & & & 111 \\
\hline & & & 103.6743 & 192.9658 & & & & & & & 297 \\
\hline & & & & 84.28392 & & 268.4594 & & & & & 353 \\
\hline & & & & & 329.6813 & 606.0012 & & & & & 936 \\
\hline & & & & & 141.5776 & & & & & & 142 \\
\hline & & & & & & & 118.6776 & & & & 119 \\
\hline & & & & & & & & 110.927 & & & 111 \\
\hline & & & & & & & & & 103.6743 & 1482.217 & 1586 \\
\hline Watercraft & 10.41006 & 2.44933 & 2.289186 & 6.121828 & 40.7497 & 19.30857 & 10.41006 & 2.44933 & 2.289186 & 32.72816 & 129 \\
\hline Cold & 2.878013 & 0.14028 & 0.131108 & 0.350613 & 9.670237 & 1.105853 & 2.878013 & 0.14028 & 0.131108 & 1.874427 & 19 \\
\hline Other & 4.038921 & 0.500248 & 0.46754 & 1.250314 & 14.47261 & 3.943558 & 4.038921 & 0.500248 & 0.46754 & 6.684357 & 36 \\
\hline
\end{tabular}




\begin{tabular}{|c|c|c|c|c|c|c|c|c|c|c|c|}
\hline & $\mathrm{f} 2$ & $\mathrm{f3}$ & $\mathrm{f} 4$ & $\mathrm{fP}$ & fC (female w/calf) & $f B$ & $\mathrm{~m} 2$ & $\mathrm{~m} 3$ & $\mathrm{~m} 4$ & $\mathrm{ma}$ & \\
\hline \multirow[t]{10}{*}{ Starting population vector: } & 142 & 119 & 111 & 297 & 353 & 936 & 142 & 119 & 111 & 1586 & 4441 \\
\hline & & & & & & & & & & & Eight Year \\
\hline & & & & & 147.3762 & & & & & & 147 \\
\hline & 123.5406 & & & & & & & & & & 124 \\
\hline & & & 107.9209 & 200.8664 & & & & & & & 309 \\
\hline & & & & 87.73476 & & 279.4699 & & & & & 367 \\
\hline & & & & & 343.184 & 630.8556 & & & & & 974 \\
\hline & & & & & 147.3762 & & & & & & 147 \\
\hline & & & & & & & 123.5406 & & & & 124 \\
\hline & & & & & & & & & 107.9209 & 1542.913 & 1651 \\
\hline Watercraft & 10.83662 & 2.549453 & 2.382953 & 6.372474 & 42.41869 & 20.10049 & 10.83662 & 2.549453 & 2.382953 & 34.06838 & 134 \\
\hline Cold & 2.995943 & 0.146014 & 0.136478 & 0.364968 & 10.0663 & 1.151208 & 2.995943 & 0.146014 & 0.136478 & 1.951185 & 20 \\
\hline Other & 4.204421 & 0.520697 & 0.486691 & 1.301506 & 15.06536 & 4.105298 & 4.204421 & 0.520697 & 0.486691 & 6.958081 & 38 \\
\hline
\end{tabular}




\begin{tabular}{|c|c|c|c|c|c|c|c|c|c|c|c|}
\hline & $\mathrm{f} 2$ & $\mathrm{f3}$ & f4 & $\mathrm{fP}$ & $\mathrm{fC}$ (female w/calf) & $\mathrm{fB}$ & $\mathrm{m} 2$ & $\mathrm{~m} 3$ & $\mathrm{~m} 4$ & ma & \\
\hline \multirow[t]{12}{*}{ Starting population vector: } & 147 & 124 & 115 & 309 & 367 & 974 & 147 & 124 & 115 & 1651 & 4623 \\
\hline & & & & & & & & & & & Ninth Year \\
\hline & & & & & 153.4181 & & & & & & 153 \\
\hline & 128.6004 & & & & & & & & & & 129 \\
\hline & & 120.1926 & & & & & & & & & 120 \\
\hline & & & 112.3325 & 209.0918 & & & & & & & 321 \\
\hline & & & & 91.32744 & & 290.9264 & & & & & 382 \\
\hline & & & & & 357.2534 & 656.7167 & & & & & 1014 \\
\hline & & & & & 153.4181 & & & & & & 153 \\
\hline & & & & & & & 128.6004 & & & & 129 \\
\hline & & & & & & & & 120.1926 & & & 120 \\
\hline & & & & & & & & & 112.3325 & 1606.097 & 1718 \\
\hline Watercraft & 11.28045 & 2.65392 & 2.480363 & 6.633422 & 44.15772 & 20.92448 & 11.28045 & 2.65392 & 2.480363 & 35.4635 & 140 \\
\hline Cold & 3.118648 & 0.151997 & 0.142057 & 0.379913 & 10.47899 & 1.1984 & 3.118648 & 0.151997 & 0.142057 & 2.031087 & 21 \\
\hline Other & 4.376621 & 0.542033 & 0.506586 & 1.354801 & 15.68299 & 4.273589 & 4.376621 & 0.542033 & 0.506586 & 7.243019 & 39 \\
\hline
\end{tabular}




\begin{tabular}{|c|c|c|c|c|c|c|c|c|c|c|c|}
\hline & $\mathrm{f} 2$ & f3 & $\mathrm{f} 4$ & $\mathrm{fP}$ & $\mathrm{fC}$ (female w/calf) & $f B$ & $\mathrm{~m} 2$ & $\mathrm{~m} 3$ & $\mathrm{~m} 4$ & $\mathrm{ma}$ & \\
\hline \multirow[t]{12}{*}{ Starting population vector: } & 153 & 129 & 120 & 321 & 382 & 1014 & 153 & 129 & 120 & 1718 & 4812 \\
\hline & & & & & & & & & & & Tenth Year \\
\hline & & & & & 159.7057 & & & & & & 160 \\
\hline & 133.8726 & & & & & & & & & & 134 \\
\hline & & 125.1154 & & & & & & & & & 125 \\
\hline & & & 116.9354 & 217.6487 & & & & & & & 335 \\
\hline & & & & 95.06494 & & 302.8529 & & & & & 398 \\
\hline & & & & & 371.8948 & 683.6386 & & & & & 1056 \\
\hline & & & & & 159.7057 & & & & & & 160 \\
\hline & & & & & & & 133.8726 & & & & 134 \\
\hline & & & & & & & & 125.1154 & & & 125 \\
\hline & & & & & & & & & 116.9354 & 1671.86 & 1789 \\
\hline Watercraft & 11.74292 & 2.762617 & 2.581999 & 6.90489 & 45.96744 & 21.78228 & 11.74292 & 2.762617 & 2.581999 & 36.91559 & 146 \\
\hline Cold & 3.246502 & 0.158222 & 0.147878 & 0.395461 & 10.90845 & 1.247528 & 3.246502 & 0.158222 & 0.147878 & 2.114252 & 22 \\
\hline Other & 4.556049 & 0.564233 & 0.527344 & 1.410246 & 16.32573 & 4.448784 & 4.556049 & 0.564233 & 0.527344 & 7.539591 & 41 \\
\hline
\end{tabular}

\section{Appendices 11}




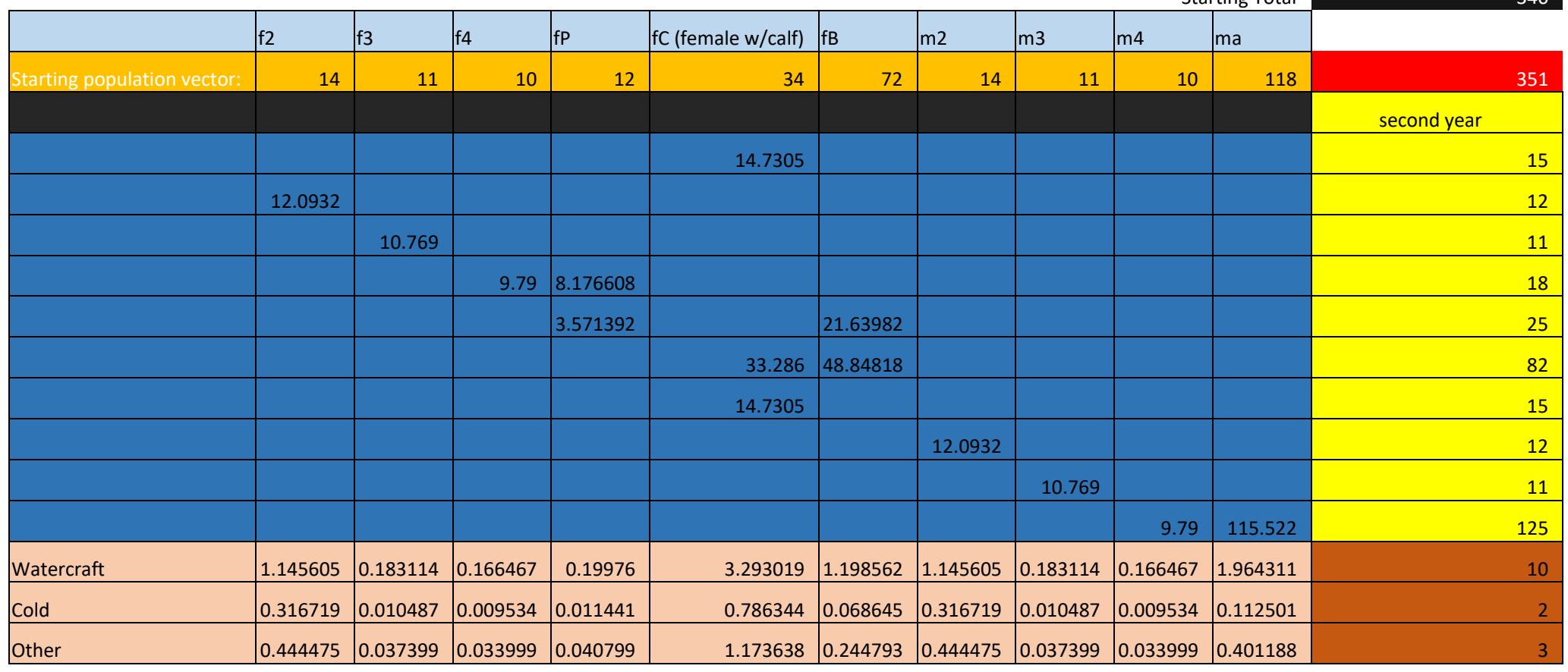




\begin{tabular}{|c|c|c|c|c|c|c|c|c|c|c|c|}
\hline & $\mathrm{f} 2$ & f3 & f4 & $\mathrm{fP}$ & $\mathrm{fC}$ (female w/calf) & $f B$ & $\mathrm{~m} 2$ & m3 & $\mathrm{m} 4$ & ma & \\
\hline \multirow[t]{12}{*}{ Starting population vector: } & 15 & 12 & 11 & 18 & 25 & 82 & 15 & 12 & 11 & 125 & 365 \\
\hline & & & & & & & & & & & second year \\
\hline & & & & & 10.53324 & & & & & & 11 \\
\hline & 12.85383 & & & & & & & & & & 13 \\
\hline & & 11.76547 & & & & & & & & & 12 \\
\hline & & & 10.47716 & 12.16588 & & & & & & & 23 \\
\hline & & & & 5.313833 & & 24.53186 & & & & & 30 \\
\hline & & & & & 24.52798 & 55.37648 & & & & & 80 \\
\hline & & & & & 10.53324 & & & & & & 11 \\
\hline & & & & & & & 12.85383 & & & & 13 \\
\hline & & & & & & & & 11.76547 & & & 12 \\
\hline & & & & & & & & & 10.47716 & 121.916 & 132 \\
\hline Watercraft & 1.127501 & 0.259788 & 0.231341 & 0.385962 & 3.031741 & 1.764421 & 1.127501 & 0.259788 & 0.231341 & 2.691974 & 11 \\
\hline Cold & 0.311714 & 0.014879 & 0.01325 & 0.022105 & 0.719457 & 0.101053 & 0.311714 & 0.014879 & 0.01325 & 0.154176 & 2 \\
\hline Other & 0.437451 & 0.053059 & 0.047249 & 0.078828 & 1.076749 & 0.360363 & 0.437451 & 0.053059 & 0.047249 & 0.549805 & 3 \\
\hline
\end{tabular}




\begin{tabular}{|c|c|c|c|c|c|c|c|c|c|c|c|}
\hline & $\mathrm{f} 2$ & $\mathrm{f3}$ & $\mathrm{f} 4$ & $\mathrm{fP}$ & fC (female w/calf) & $f B$ & $\mathrm{~m} 2$ & m3 & $\mathrm{m} 4$ & ma & \\
\hline \multirow[t]{12}{*}{ Starting population vector: } & 11 & 13 & 12 & 23 & 30 & 80 & 11 & 13 & 12 & 132 & 379 \\
\hline & & & & & & & & & & & Third year \\
\hline & & & & & 12.46953 & & & & & & 12 \\
\hline & 9.191308 & & & & & & & & & & 9 \\
\hline & & 12.5055 & & & & & & & & & 13 \\
\hline & & & 11.44663 & 15.33247 & & & & & & & 27 \\
\hline & & & & 6.696942 & & 23.86589 & & & & & 31 \\
\hline & & & & & 29.03688 & 53.87317 & & & & & 83 \\
\hline & & & & & 12.46953 & & & & & & 12 \\
\hline & & & & & & & 9.191308 & & & & 9 \\
\hline & & & & & & & & 12.5055 & & & 13 \\
\hline & & & & & & & & & 11.44663 & 128.8053 & 140 \\
\hline Watercraft & 0.806235 & 0.276128 & 0.252748 & 0.486422 & 3.589055 & 1.716521 & 0.806235 & 0.276128 & 0.252748 & 2.844093 & 11 \\
\hline Cold & 0.222895 & 0.015815 & 0.014476 & 0.027859 & 0.851712 & 0.09831 & 0.222895 & 0.015815 & 0.014476 & 0.162889 & 2 \\
\hline Other & 0.312805 & 0.056396 & 0.051621 & 0.099346 & 1.274684 & 0.35058 & 0.312805 & 0.056396 & 0.051621 & 0.580874 & 3 \\
\hline
\end{tabular}




\begin{tabular}{|c|c|c|c|c|c|c|c|c|c|c|c|}
\hline & $\mathrm{f} 2$ & $\mathrm{f3}$ & $\mathrm{f} 4$ & $\mathrm{fP}$ & $\mathrm{fC}$ (female w/calf) & $f B$ & $\mathrm{~m} 2$ & m3 & $\mathrm{m} 4$ & $\mathrm{ma}$ & \\
\hline \multirow[t]{12}{*}{ Starting population vector: } & 12 & 9 & 13 & 27 & 31 & 83 & 12 & 9 & 13 & 140 & 395 \\
\hline & & & & & & & & & & & Forth year \\
\hline & & & & & 12.76915 & & & & & & 13 \\
\hline & 10.88091 & & & & & & & & & & 11 \\
\hline & & 8.942223 & & & & & & & & & 9 \\
\hline & & & 12.1666 & 18.13316 & & & & & & & 30 \\
\hline & & & & 7.92023 & & 24.7636 & & & & & 33 \\
\hline & & & & & 29.73458 & 55.89959 & & & & & 86 \\
\hline & & & & & 12.76915 & & & & & & 13 \\
\hline & & & & & & & 10.88091 & & & & 11 \\
\hline & & & & & & & & 8.942223 & & & 9 \\
\hline & & & & & & & & & 12.1666 & 136.4512 & 149 \\
\hline Watercraft & 0.954442 & 0.197449 & 0.268645 & 0.575273 & 3.675293 & 1.781088 & 0.954442 & 0.197449 & 0.268645 & 3.012917 & 12 \\
\hline Cold & 0.26387 & 0.011308 & 0.015386 & 0.032947 & 0.872177 & 0.102008 & 0.26387 & 0.011308 & 0.015386 & 0.172558 & 2 \\
\hline Other & 0.370307 & 0.040327 & 0.054868 & 0.117493 & 1.305312 & 0.363767 & 0.370307 & 0.040327 & 0.054868 & 0.615354 & 3 \\
\hline
\end{tabular}


Undergraduate Journal of Mathematical M odeling: One + Two, Vol. 9, Iss. 2 [2019], Art. 5

\begin{tabular}{|c|c|c|c|c|c|c|c|c|c|c|c|}
\hline & $\mathrm{f} 2$ & $\mathrm{f3}$ & f4 & fP & fC (female w/calf) & $f B$ & $\mathrm{~m} 2$ & m3 & m4 & ma & \\
\hline \multirow[t]{10}{*}{ Starting population vector: } & 13 & 11 & 9 & 30 & 33 & 86 & 13 & 11 & 9 & 149 & 412 \\
\hline & & & & & & & & & & & Fifth Year \\
\hline & & & & & 13.6553 & & & & & & 14 \\
\hline & 11.14236 & & & & & & & & & & 11 \\
\hline & & 10.58604 & & & & & & & & & 11 \\
\hline & & & & 8.961504 & & 25.57724 & & & & & 35 \\
\hline & & & & & 31.7981 & 57.73624 & & & & & 90 \\
\hline & & & & & 13.6553 & & & & & & 14 \\
\hline & & & & & & & 11.14236 & & & & 11 \\
\hline & & & & & & & & 10.58604 & & & 11 \\
\hline Watercraft & 0.977375 & 0.233746 & 0.192098 & 0.650904 & 3.930351 & 1.839608 & 0.977375 & 0.233746 & 0.192098 & 3.192632 & 12 \\
\hline Cold & 0.27021 & 0.013387 & 0.011002 & 0.037279 & 0.932704 & 0.105359 & 0.27021 & 0.013387 & 0.011002 & 0.18285 & 2 \\
\hline Other & 0.379205 & 0.04774 & 0.039234 & 0.13294 & 1.395898 & 0.375719 & 0.379205 & 0.04774 & 0.039234 & 0.652059 & 3 \\
\hline
\end{tabular}




\begin{tabular}{|c|c|c|c|c|c|c|c|c|c|c|c|}
\hline & $\mathrm{f} 2$ & f3 & $\mathrm{f} 4$ & $\mathrm{fP}$ & $\mathrm{fC}$ (female w/calf) & $\mathrm{fB}$ & $\mathrm{m} 2$ & $\mathrm{~m} 3$ & $\mathrm{~m} 4$ & $\mathrm{ma}$ & \\
\hline \multirow[t]{12}{*}{ Starting population vector: } & 14 & 11 & 11 & 29 & 35 & 90 & 14 & 11 & 11 & 153 & 429 \\
\hline & & & & & & & & & & & Sixth Year \\
\hline & & & & & 14.43029 & & & & & & 14 \\
\hline & 11.91562 & & & & & & & & & & 12 \\
\hline & & 10.8404 & & & & & & & & & 11 \\
\hline & & & 10.29916 & 19.78396 & & & & & & & 30 \\
\hline & & & & 8.641272 & & 26.74214 & & & & & 35 \\
\hline & & & & & 33.60274 & 60.36581 & & & & & 94 \\
\hline & & & & & 14.43029 & & & & & & 14 \\
\hline & & & & & & & 11.91562 & & & & 12 \\
\hline & & & & & & & & 10.8404 & & & 11 \\
\hline & & & & & & & & & 10.29916 & 149.1359 & 159 \\
\hline Watercraft & 1.045203 & 0.239362 & 0.227411 & 0.627645 & 4.153411 & 1.923392 & 1.045203 & 0.239362 & 0.227411 & 3.293004 & 13 \\
\hline Cold & 0.288962 & 0.013709 & 0.013024 & 0.035947 & 0.985638 & 0.110158 & 0.288962 & 0.013709 & 0.013024 & 0.188599 & 2 \\
\hline Other & 0.405521 & 0.048887 & 0.046446 & 0.128189 & 1.47512 & 0.392831 & 0.405521 & 0.048887 & 0.046446 & 0.672559 & 4 \\
\hline
\end{tabular}




\begin{tabular}{|c|c|c|c|c|c|c|c|c|c|c|c|}
\hline & $\mathrm{f} 2$ & $\mathrm{f3}$ & $\mathrm{f} 4$ & $\mathrm{fP}$ & $\mathrm{fC}$ (female w/calf) & $\mathrm{fB}$ & $\mathrm{m} 2$ & $\mathrm{~m} 3$ & $\mathrm{~m} 4$ & $\mathrm{ma}$ & \\
\hline \multirow[t]{10}{*}{ Starting population vector: } & 14 & 12 & 11 & 30 & 35 & 94 & 14 & 12 & 11 & 159 & 446 \\
\hline & & & & & & & & & & & Seventh year \\
\hline & & & & & 14.78319 & & & & & & 15 \\
\hline & 12.59187 & & & & & & & & & & 13 \\
\hline & & 11.5927 & & & & & & & & & 12 \\
\hline & & & & 8.897432 & & 28.06656 & & & & & 37 \\
\hline & & & & & 34.42452 & 63.35545 & & & & & 98 \\
\hline & & & & & 14.78319 & & & & & & 15 \\
\hline & & & & & & & 12.59187 & & & & 13 \\
\hline & & & & & & & & 11.5927 & & & 12 \\
\hline Watercraft & 1.104522 & 0.255973 & 0.232875 & 0.646251 & 4.254986 & 2.018648 & 1.104522 & 0.255973 & 0.232875 & 3.425012 & 14 \\
\hline Cold & 0.305361 & 0.01466 & 0.013337 & 0.037012 & 1.009743 & 0.115613 & 0.305361 & 0.01466 & 0.013337 & 0.196159 & 2 \\
\hline Other & 0.428535 & 0.05228 & 0.047562 & 0.131989 & 1.511195 & 0.412286 & 0.428535 & 0.05228 & 0.047562 & 0.69952 & 4 \\
\hline
\end{tabular}




\begin{tabular}{|c|c|c|c|c|c|c|c|c|c|c|c|}
\hline & $\mathrm{f} 2$ & $\mathrm{f3}$ & $f 4$ & $\mathrm{fP}$ & fC (female w/calf) & $f B$ & $\mathrm{~m} 2$ & m3 & $\mathrm{m} 4$ & ma & \\
\hline \multirow{11}{*}{ Starting population vector: } & 15 & 13 & 12 & 31 & 37 & 98 & 15 & 13 & 12 & 166 & 464 \\
\hline & & & & & & & & & & & Eight Year \\
\hline & & & & & 15.44355 & & & & & & 15 \\
\hline & 12.89981 & & & & & & & & & & 13 \\
\hline & & 12.25063 & & & & & & & & & 12 \\
\hline & & & 11.27854 & 20.93513 & & & & & & & 32 \\
\hline & & & & 9.144081 & & 29.20495 & & & & & 38 \\
\hline & & & & & 35.96226 & 65.92519 & & & & & 102 \\
\hline & & & & & 15.44355 & & & & & & 15 \\
\hline & & & & & & & 12.89981 & & & & 13 \\
\hline & & & & & & & & 12.25063 & & & 12 \\
\hline Watercraft & 1.131534 & 0.270501 & 0.249036 & 0.664166 & 4.445056 & 2.100526 & 1.131534 & 0.270501 & 0.249036 & 3.558758 & 14 \\
\hline Cold & 0.312829 & 0.015492 & 0.014263 & 0.038039 & 1.054848 & 0.120303 & 0.312829 & 0.015492 & 0.014263 & 0.203819 & 2 \\
\hline Other & 0.439016 & 0.055247 & 0.050863 & 0.135648 & 1.5787 & 0.429009 & 0.439016 & 0.055247 & 0.050863 & 0.726836 & 4 \\
\hline
\end{tabular}


Undergraduate Journal of Mathematical M odeling: One + Two, Vol. 9, Iss. 2 [2019], Art. 5

\begin{tabular}{|c|c|c|c|c|c|c|c|c|c|c|}
\hline & $\mathrm{f} 2$ & $\mathrm{f3}$ & $\mathrm{f} 4$ & $\mathrm{fP}$ & $\mathrm{fC}$ (female w/calf) & $\mathrm{fB}$ & $\mathrm{m} 2$ & m3 & m4 & ma \\
\hline \multirow[t]{12}{*}{ Starting population vector: } & 15 & 13 & 12 & 32 & 38 & 102 & 15 & 13 & 12 & 172 \\
\hline & & & & & & & & & & \\
\hline & & & & & 16.02223 & & & & & \\
\hline & 13.47605 & & & & & & & & & \\
\hline & & 12.55023 & & & & & & & & \\
\hline & & & 11.91864 & 21.81312 & & & & & & \\
\hline & & & & 9.527568 & & 30.43177 & & & & \\
\hline & & & & & 37.30977 & 68.69453 & & & & \\
\hline & & & & & 16.02223 & & & & & \\
\hline & & & & & & & 13.47605 & & & \\
\hline & & & & & & & & 12.55023 & & \\
\hline & & & & & & & & & 11.91864 & 167.7768 \\
\hline Watercraft & 1.182079 & 0.277116 & 0.26317 & 0.69202 & 4.611613 & 2.188764 & 1.182079 & 0.277116 & 0.26317 & 3.704604 \\
\hline Cold & 0.326803 & 0.015871 & 0.015072 & 0.039634 & 1.094373 & 0.125356 & 0.326803 & 0.015871 & 0.015072 & 0.212172 \\
\hline Other & 0.458626 & 0.056598 & 0.05375 & 0.141337 & 1.637854 & 0.44703 & 0.458626 & 0.056598 & 0.05375 & 0.756623 \\
\hline
\end{tabular}




\begin{tabular}{|c|c|c|c|c|c|c|c|c|c|c|c|}
\hline & $\mathrm{f} 2$ & $\mathrm{f3}$ & $\mathrm{f} 4$ & $\mathrm{fP}$ & $\mathrm{fC}$ (female w/calf) & $f B$ & $\mathrm{~m} 2$ & $\mathrm{~m} 3$ & $\mathrm{~m} 4$ & $\mathrm{ma}$ & \\
\hline \multirow[t]{12}{*}{ Starting population vector: } & 16 & 13 & 13 & 34 & 40 & 106 & 16 & 13 & 13 & 180 & 503 \\
\hline & & & & & & & & & & & Tenth Year \\
\hline & & & & & 16.69501 & & & & & & 17 \\
\hline & 13.98099 & & & & & & & & & & 14 \\
\hline & & 13.11084 & & & & & & & & & 13 \\
\hline & & & 12.21012 & 22.84106 & & & & & & & 35 \\
\hline & & & & 9.976557 & & 31.6614 & & & & & 42 \\
\hline & & & & & 38.87644 & 71.47019 & & & & & 110 \\
\hline & & & & & 16.69501 & & & & & & 17 \\
\hline & & & & & & & 13.98099 & & & & 14 \\
\hline & & & & & & & & 13.11084 & & & 13 \\
\hline & & & & & & & & & 12.21012 & 174.8256 & 187 \\
\hline Watercraft & 1.226372 & 0.289495 & 0.269606 & 0.724631 & 4.805258 & 2.277202 & 1.226372 & 0.289495 & 0.269606 & 3.860247 & 15 \\
\hline Cold & 0.339049 & 0.01658 & 0.015441 & 0.041502 & 1.140327 & 0.130421 & 0.339049 & 0.01658 & 0.015441 & 0.221086 & 2 \\
\hline Other & 0.475811 & 0.059126 & 0.055064 & 0.147998 & 1.706629 & 0.465093 & 0.475811 & 0.059126 & 0.055064 & 0.788412 & 4 \\
\hline
\end{tabular}




\begin{tabular}{|c|c|c|c|c|c|c|c|c|c|c|c|}
\hline & & & & & & & & & & & \\
\hline & $\mathrm{f} 2$ & $\mathrm{f3}$ & f4 & $\mathrm{fP}$ & fC (female w/calf) & $\mathrm{fB}$ & $\mathrm{m} 2$ & $\mathrm{~m} 3$ & m4 & $\mathrm{ma}$ & \\
\hline \multirow[t]{12}{*}{ Starting population vector: } & 24 & 21 & 19 & 41 & 54 & 119 & 24 & 21 & 19 & 214 & 640 \\
\hline & & & & & & & & & & & First year \\
\hline & & & & & 25.0047 & & & & & & 25 \\
\hline & 22.6272 & & & & & & & & & & 23 \\
\hline & & 20.538 & & & & & & & & & 21 \\
\hline & & & 18.582 & 27.90821 & & & & & & & 46 \\
\hline & & & & 12.18979 & & 35.72927 & & & & & 48 \\
\hline & & & & & 52.812 & 80.65273 & & & & & 133 \\
\hline & & & & & 25.0047 & & & & & & 25 \\
\hline & & & & & & & 22.6272 & & & & 23 \\
\hline & & & & & & & & 20.538 & & & 21 \\
\hline & & & & & & & & & 18.582 & 209.292 & 228 \\
\hline Watercraft & 0.824778 & 0.366227 & 0.331349 & 0.715015 & 3.33928 & 2.075289 & 0.824778 & 0.366227 & 0.331349 & 3.732032 & 13 \\
\hline Cold & 0.228022 & 0.020975 & 0.018977 & 0.040951 & 0.716774 & 0.118857 & 0.228022 & 0.020975 & 0.018977 & 0.213743 & 2 \\
\hline Other & 0.32 & 0.074798 & 0.067674 & 0.146034 & 1.122546 & 0.423854 & 0.32 & 0.074798 & 0.067674 & 0.762225 & 3 \\
\hline
\end{tabular}




\begin{tabular}{|c|c|c|c|c|c|c|c|c|c|c|c|}
\hline & $\mathrm{f} 2$ & $\mathrm{f3}$ & $\mathrm{f} 4$ & $\mathrm{fP}$ & fC (female w/calf) & $\mathrm{fB}$ & $\mathrm{m} 2$ & m3 & $\mathrm{m} 4$ & ma & \\
\hline \multirow[t]{12}{*}{ Starting population vector: } & 25 & 23 & 21 & 46 & 48 & 133 & 25 & 23 & 21 & 228 & 665 \\
\hline & & & & & & & & & & & second year \\
\hline & & & & & 20.02059 & & & & & & 20 \\
\hline & 21.8191 & & & & & & & & & & 22 \\
\hline & & 22.014 & & & & & & & & & 22 \\
\hline & & & 19.98142 & 31.48031 & & & & & & & 51 \\
\hline & & & & 13.75002 & & 39.86328 & & & & & 54 \\
\hline & & & & & 46.62046 & 89.98455 & & & & & 137 \\
\hline & & & & & 20.02059 & & & & & & 20 \\
\hline & & & & & & & 21.8191 & & & & 22 \\
\hline & & & & & & & & 22.014 & & & 22 \\
\hline & & & & & & & & & 19.98142 & 221.6986 & 242 \\
\hline Watercraft & 1.913908 & 0.486081 & 0.441201 & 0.998711 & 5.762445 & 2.867112 & 1.913908 & 0.486081 & 0.441201 & 4.895228 & 20 \\
\hline Cold & 0.529128 & 0.027839 & 0.025269 & 0.057199 & 1.367475 & 0.164207 & 0.529128 & 0.027839 & 0.025269 & 0.280362 & 3 \\
\hline Other & 0.742563 & 0.099277 & 0.09011 & 0.203975 & 2.046582 & 0.585575 & 0.742563 & 0.099277 & 0.09011 & 0.999795 & 6 \\
\hline
\end{tabular}




\begin{tabular}{|c|c|c|c|c|c|c|c|c|c|c|c|}
\hline & $\mathrm{f} 2$ & $\mathrm{f3}$ & $\mathrm{f} 4$ & $\mathrm{fP}$ & fC (female w/calf) & $\mathrm{fB}$ & $\mathrm{m} 2$ & m3 & $\mathrm{m} 4$ & $\mathrm{ma}$ & \\
\hline \multirow[t]{12}{*}{ Starting population vector: } & 20 & 22 & 22 & 51 & 54 & 137 & 20 & 22 & 22 & 242 & 691 \\
\hline & & & & & & & & & & & Third year \\
\hline & & & & & 22.39964 & & & & & & 22 \\
\hline & 17.46996 & & & & & & & & & & 17 \\
\hline & & 21.2278 & & & & & & & & & 21 \\
\hline & & & 21.41742 & 34.84671 & & & & & & & 56 \\
\hline & & & & 15.2204 & & 40.80122 & & & & & 56 \\
\hline & & & & & 52.16038 & 92.10179 & & & & & 144 \\
\hline & & & & & 22.39964 & & & & & & 22 \\
\hline & & & & & & & 17.46996 & & & & 17 \\
\hline & & & & & & & & 21.2278 & & & 21 \\
\hline & & & & & & & & & 21.41742 & 235.1305 & 257 \\
\hline Watercraft & 1.532414 & 0.468722 & 0.472909 & 1.10551 & 6.447197 & 2.934572 & 1.532414 & 0.468722 & 0.472909 & 5.191812 & 21 \\
\hline Cold & 0.423658 & 0.026845 & 0.027085 & 0.063315 & 1.529972 & 0.168071 & 0.423658 & 0.026845 & 0.027085 & 0.297349 & 3 \\
\hline Other & 0.59455 & 0.095731 & 0.096586 & 0.225788 & 2.289778 & 0.599353 & 0.59455 & 0.095731 & 0.096586 & 1.060369 & 6 \\
\hline
\end{tabular}




\begin{tabular}{|c|c|c|c|c|c|c|c|c|c|c|c|}
\hline & $\mathrm{f} 2$ & $\mathrm{f3}$ & $\mathrm{f} 4$ & $\mathrm{fP}$ & fC (female w/calf) & $\mathrm{fB}$ & $\mathrm{m} 2$ & m3 & $\mathrm{m} 4$ & $\mathrm{ma}$ & \\
\hline \multirow[t]{12}{*}{ Starting population vector: } & 22 & 17 & 21 & 56 & 56 & 144 & 22 & 17 & 21 & 257 & 720 \\
\hline & & & & & & & & & & & Forth year \\
\hline & & & & & 23.40584 & & & & & & 23 \\
\hline & 19.54592 & & & & & & & & & & 20 \\
\hline & & 16.99653 & & & & & & & & & 17 \\
\hline & & & 20.65253 & 38.09861 & & & & & & & 59 \\
\hline & & & & 16.64077 & & 43.08827 & & & & & 60 \\
\hline & & & & & 54.50344 & 97.2644 & & & & & 152 \\
\hline & & & & & 23.40584 & & & & & & 23 \\
\hline & & & & & & & 19.54592 & & & & 20 \\
\hline & & & & & & & & 16.99653 & & & 17 \\
\hline & & & & & & & & & 20.65253 & 249.5955 & 270 \\
\hline Watercraft & 1.714511 & 0.375293 & 0.456019 & 1.208676 & 6.736807 & 3.099064 & 1.714511 & 0.375293 & 0.456019 & 5.511206 & 22 \\
\hline Cold & 0.474002 & 0.021494 & 0.026117 & 0.069224 & 1.598699 & 0.177492 & 0.474002 & 0.021494 & 0.026117 & 0.315641 & 3 \\
\hline Other & 0.665201 & 0.076649 & 0.093137 & 0.246858 & 2.392635 & 0.632949 & 0.665201 & 0.076649 & 0.093137 & 1.125601 & 6 \\
\hline
\end{tabular}


Undergraduate Journal of M athematical M odeling: One + Two, Vol. 9, Iss. 2 [2019], Art. 5

\begin{tabular}{|c|c|c|c|c|c|c|c|c|c|c|c|}
\hline & $\mathrm{f} 2$ & f3 & $\mathrm{f} 4$ & $\mathrm{fP}$ & $\mathrm{fC}$ (female w/calf) & $\mathrm{fB}$ & $\mathrm{m} 2$ & m3 & $\mathrm{m} 4$ & $\mathrm{ma}$ & \\
\hline \multirow[t]{12}{*}{ Starting population vector: } & 23 & 20 & 17 & 59 & 60 & 152 & 23 & 20 & 17 & 270 & 750 \\
\hline & & & & & & & & & & & Fifth Year \\
\hline & & & & & 24.95479 & & & & & & 25 \\
\hline & 20.42393 & & & & & & & & & & 20 \\
\hline & & 19.01623 & & & & & & & & & 19 \\
\hline & & & 16.53592 & 39.78265 & & & & & & & 56 \\
\hline & & & & 17.37633 & & 45.33006 & & & & & 63 \\
\hline & & & & & 58.11038 & 102.3249 & & & & & 160 \\
\hline & & & & & 24.95479 & & & & & & 25 \\
\hline & & & & & & & 20.42393 & & & & 20 \\
\hline & & & & & & & & 19.01623 & & & 19 \\
\hline & & & & & & & & & 16.53592 & 262.9243 & 279 \\
\hline Watercraft & 1.791528 & 0.419889 & 0.365122 & 1.262102 & 7.182637 & 3.260302 & 1.791528 & 0.419889 & 0.365122 & 5.805514 & 23 \\
\hline Cold & 0.495294 & 0.024048 & 0.020912 & 0.072284 & 1.704498 & 0.186726 & 0.495294 & 0.024048 & 0.020912 & 0.332497 & 3 \\
\hline Other & 0.695082 & 0.085758 & 0.074572 & 0.25777 & 2.550975 & 0.66588 & 0.695082 & 0.085758 & 0.074572 & 1.18571 & 6 \\
\hline
\end{tabular}




\begin{tabular}{|c|c|c|c|c|c|c|c|c|c|c|c|}
\hline & $\mathrm{f} 2$ & $\mathrm{f3}$ & $\mathrm{f} 4$ & $\mathrm{fP}$ & fC (female w/calf) & $\mathrm{fB}$ & $\mathrm{m} 2$ & m3 & $\mathrm{m} 4$ & ma & \\
\hline \multirow[t]{12}{*}{ Starting population vector: } & 25 & 20 & 19 & 56 & 63 & 160 & 25 & 20 & 19 & 279 & 781 \\
\hline & & & & & & & & & & & Sixth Year \\
\hline & & & & & 26.19873 & & & & & & 26 \\
\hline & 21.77555 & & & & & & & & & & 22 \\
\hline & & 19.87044 & & & & & & & & & 20 \\
\hline & & & 18.50089 & 38.13547 & & & & & & & 57 \\
\hline & & & & 16.65687 & & 47.91885 & & & & & 65 \\
\hline & & & & & 61.00705 & 108.1686 & & & & & 169 \\
\hline & & & & & 26.19873 & & & & & & 26 \\
\hline & & & & & & & 21.77555 & & & & 22 \\
\hline & & & & & & & & 19.87044 & & & 20 \\
\hline & & & & & & & & & 18.50089 & 271.8868 & 290 \\
\hline Watercraft & 1.910088 & 0.43875 & 0.40851 & 1.209845 & 7.540675 & 3.446497 & 1.910088 & 0.43875 & 0.40851 & 6.003412 & 24 \\
\hline Cold & 0.528072 & 0.025128 & 0.023396 & 0.069291 & 1.789464 & 0.19739 & 0.528072 & 0.025128 & 0.023396 & 0.343831 & 4 \\
\hline Other & 0.741081 & 0.08961 & 0.083434 & 0.247097 & 2.678135 & 0.703908 & 0.741081 & 0.08961 & 0.083434 & 1.226129 & 7 \\
\hline
\end{tabular}




\begin{tabular}{|c|c|c|c|c|c|c|c|c|c|c|}
\hline & $\mathrm{f} 2$ & $\mathrm{f3}$ & $\mathrm{f} 4$ & $\mathrm{fP}$ & $\mathrm{fC}$ (female w/calf) & $f B$ & $\mathrm{~m} 2$ & m3 & $\mathrm{m} 4$ & $\mathrm{ma}$ \\
\hline \multirow[t]{12}{*}{ Starting population vector: } & 26 & 22 & 20 & 57 & 65 & 169 & 26 & 22 & 20 & 290 \\
\hline & & & & & & & & & & \\
\hline & & & & & 26.97974 & & & & & \\
\hline & 22.86101 & & & & & & & & & \\
\hline & & 21.18543 & & & & & & & & \\
\hline & & & 19.33195 & 38.35065 & & & & & & \\
\hline & & & & 16.75086 & & 50.52943 & & & & \\
\hline & & & & & 62.82572 & 114.0616 & & & & \\
\hline & & & & & 26.97974 & & & & & \\
\hline & & & & & & & 22.86101 & & & \\
\hline & & & & & & & & 21.18543 & & \\
\hline & & & & & & & & & 19.33195 & 282.5182 \\
\hline Watercraft & 2.005301 & 0.467786 & 0.42686 & 1.216672 & 7.765468 & 3.63426 & 2.005301 & 0.467786 & 0.42686 & 6.238159 \\
\hline Cold & 0.554395 & 0.026791 & 0.024447 & 0.069682 & 1.842809 & 0.208144 & 0.554395 & 0.026791 & 0.024447 & 0.357276 \\
\hline Other & 0.778022 & 0.09554 & 0.087181 & 0.248491 & 2.757973 & 0.742256 & 0.778022 & 0.09554 & 0.087181 & 1.274073 \\
\hline
\end{tabular}




\begin{tabular}{|c|c|c|c|c|c|c|c|c|c|c|c|}
\hline & $\mathrm{f} 2$ & $\mathrm{f3}$ & $\mathrm{f} 4$ & $\mathrm{fP}$ & fC (female w/calf) & $\mathrm{fB}$ & $\mathrm{m} 2$ & m3 & $\mathrm{m} 4$ & $\mathrm{ma}$ & \\
\hline \multirow[t]{12}{*}{ Starting population vector: } & 27 & 23 & 21 & 58 & 67 & 177 & 27 & 23 & 21 & 302 & 846 \\
\hline & & & & & & & & & & & Eight Year \\
\hline & & & & & 28.10971 & & & & & & 28 \\
\hline & 23.54252 & & & & & & & & & & 24 \\
\hline & & 22.24148 & & & & & & & & & 22 \\
\hline & & & 20.61131 & 39.05911 & & & & & & & 60 \\
\hline & & & & 17.0603 & & 52.83274 & & & & & 70 \\
\hline & & & & & 65.457 & 119.2609 & & & & & 185 \\
\hline & & & & & 28.10971 & & & & & & 28 \\
\hline & & & & & & & 23.54252 & & & & 24 \\
\hline & & & & & & & & 22.24148 & & & 22 \\
\hline & & & & & & & & & 20.61131 & 293.67 & 314 \\
\hline Watercraft & 2.065081 & 0.491104 & 0.455109 & 1.239148 & 8.090704 & 3.799923 & 2.065081 & 0.491104 & 0.455109 & 6.484397 & 26 \\
\hline Cold & 0.570922 & 0.028127 & 0.026065 & 0.070969 & 1.91999 & 0.217631 & 0.570922 & 0.028127 & 0.026065 & 0.371378 & 4 \\
\hline Other & 0.801216 & 0.100302 & 0.092951 & 0.253082 & 2.873483 & 0.776091 & 0.801216 & 0.100302 & 0.092951 & 1.324365 & 7 \\
\hline
\end{tabular}


Undergraduate Journal of Mathematical M odeling: One + Two, Vol. 9, Iss. 2 [2019], Art. 5

\begin{tabular}{|c|c|c|c|c|c|c|c|c|c|c|c|}
\hline & $\mathrm{f} 2$ & $\mathrm{f3}$ & $\mathrm{f} 4$ & $\mathrm{fP}$ & fC (female w/calf) & $f B$ & $\mathrm{~m} 2$ & $\mathrm{~m} 3$ & $\mathrm{~m} 4$ & $\mathrm{ma}$ & \\
\hline \multirow[t]{12}{*}{ Starting population vector: } & 28 & 24 & 22 & 60 & 70 & 185 & 28 & 24 & 22 & 314 & 881 \\
\hline & & & & & & & & & & & Ninth Year \\
\hline & & & & & 29.20131 & & & & & & 29 \\
\hline & 24.52853 & & & & & & & & & & 25 \\
\hline & & 22.90451 & & & & & & & & & 23 \\
\hline & & & 21.63874 & 40.40513 & & & & & & & 62 \\
\hline & & & & 17.64822 & & 55.17159 & & & & & 73 \\
\hline & & & & & 67.99894 & 124.5404 & & & & & 193 \\
\hline & & & & & 29.20131 & & & & & & 29 \\
\hline & & & & & & & 24.52853 & & & & 25 \\
\hline & & & & & & & & 22.90451 & & & 23 \\
\hline & & & & & & & & & 21.63874 & 305.7643 & 327 \\
\hline Watercraft & 2.151571 & 0.505744 & 0.477795 & 1.28185 & 8.404896 & 3.968141 & 2.151571 & 0.505744 & 0.477795 & 6.751445 & 27 \\
\hline Cold & 0.594833 & 0.028965 & 0.027365 & 0.073415 & 1.99455 & 0.227266 & 0.594833 & 0.028965 & 0.027365 & 0.386673 & 4 \\
\hline Other & 0.834772 & 0.103293 & 0.097584 & 0.261803 & 2.985071 & 0.810448 & 0.834772 & 0.103293 & 0.097584 & 1.378906 & 8 \\
\hline
\end{tabular}




\begin{tabular}{|c|c|c|c|c|c|c|c|c|c|c|c|}
\hline & $\mathrm{f} 2$ & $\mathrm{f3}$ & $\mathrm{f} 4$ & $\mathrm{fP}$ & $\mathrm{fC}$ (female w/calf) & $f B$ & $\mathrm{~m} 2$ & $\mathrm{~m} 3$ & $\mathrm{~m} 4$ & ma & \\
\hline \multirow[t]{12}{*}{ Starting population vector: } & 29 & 25 & 23 & 62 & 73 & 193 & 29 & 25 & 23 & 327 & 917 \\
\hline & & & & & & & & & & & Tenth Year \\
\hline & & & & & 30.42412 & & & & & & 30 \\
\hline & 25.48107 & & & & & & & & & & 25 \\
\hline & & 23.86381 & & & & & & & & & 24 \\
\hline & & & 22.2838 & 42.01228 & & & & & & & 64 \\
\hline & & & & 18.35019 & & 57.50772 & & & & & 76 \\
\hline & & & & & 70.84639 & 129.8138 & & & & & 201 \\
\hline & & & & & 30.42412 & & & & & & 30 \\
\hline & & & & & & & 25.48107 & & & & 25 \\
\hline & & & & & & & & 23.86381 & & & 24 \\
\hline & & & & & & & & & 22.2838 & 318.5304 & 341 \\
\hline Watercraft & 2.235125 & 0.526926 & 0.492039 & 1.332837 & 8.756851 & 4.136164 & 2.235125 & 0.526926 & 0.492039 & 7.033328 & 28 \\
\hline Cold & 0.617933 & 0.030178 & 0.02818 & 0.076335 & 2.078072 & 0.236889 & 0.617933 & 0.030178 & 0.02818 & 0.402817 & 4 \\
\hline Other & 0.86719 & 0.107619 & 0.100493 & 0.272217 & 3.110071 & 0.844765 & 0.86719 & 0.107619 & 0.100493 & 1.436478 & 8 \\
\hline
\end{tabular}

\section{Appendices 31}

Produced by The Berkeley Electronic Press, 2019 


\begin{tabular}{|c|c|c|c|c|c|c|c|c|c|c|c|}
\hline & $\mathrm{f} 2$ & $\mathrm{f} 3$ & $\mathrm{f} 4$ & $\mathrm{fP}$ & fC (female w/calf) & $f B$ & $\mathrm{~m} 2$ & m3 & $\mathrm{m} 4$ & ma & \\
\hline \multirow[t]{11}{*}{ Starting population vector: } & 78 & 69 & 64 & 166 & 175 & 451 & 78 & 69 & 64 & 792 & 2300 \\
\hline & & & & & & & & & & & First year \\
\hline & & & & & 82.25 & & & & & & 82 \\
\hline & 72.8754 & & & & & & & & & & 73 \\
\hline & & 67.3371 & & & & & & & & & 67 \\
\hline & & & 62.4576 & 112.7516 & & & & & & & 175 \\
\hline & & & & 49.24782 & & 135.1202 & & & & & 184 \\
\hline & & & & & 170.7825 & 305.0107 & & & & & 476 \\
\hline & & & & & 82.25 & & & & & & 82 \\
\hline & & & & & & & 72.8754 & & & & 73 \\
\hline & & & & & & & & 67.3371 & & & 67 \\
\hline Watercraft & 3.07886 & 1.318181 & 1.22266 & 3.171276 & 9.651612 & 8.615936 & 3.07886 & 1.318181 & 1.22266 & 15.13042 & 48 \\
\hline Cold & 0.851196 & 0.075496 & 0.070025 & 0.181627 & 1.935525 & 0.493457 & 0.851196 & 0.075496 & 0.070025 & 0.866559 & 5 \\
\hline Other & 1.194544 & 0.269224 & 0.249715 & 0.647697 & 3.130363 & 1.759707 & 1.194544 & 0.269224 & 0.249715 & 3.090218 & 12 \\
\hline
\end{tabular}




\begin{tabular}{|c|c|c|c|c|c|c|c|c|c|c|c|}
\hline & $\mathrm{f} 2$ & $\mathrm{f3}$ & $\mathrm{f} 4$ & $\mathrm{fP}$ & $\mathrm{fC}$ (female w/calf) & $\mathrm{fB}$ & $\mathrm{m} 2$ & m3 & $\mathrm{m} 4$ & $\mathrm{ma}$ & \\
\hline \multirow[t]{12}{*}{ Starting population vector: } & 82 & 73 & 67 & 175 & 184 & 476 & 82 & 73 & 67 & 835 & 2390 \\
\hline & & & & & & & & & & & second year \\
\hline & & & & & 77.02895 & & & & & & 77 \\
\hline & 71.77135 & & & & & & & & & & 72 \\
\hline & & 70.90048 & & & & & & & & & 71 \\
\hline & & & 65.51226 & 118.6409 & & & & & & & 184 \\
\hline & & & & 51.82015 & & 142.1101 & & & & & 194 \\
\hline & & & & & 179.3716 & 320.7892 & & & & & 500 \\
\hline & & & & & 77.02895 & & & & & & 77 \\
\hline & & & & & & & 71.77135 & & & & 72 \\
\hline & & & & & & & & 70.90048 & & & 71 \\
\hline & & & & & & & & & 65.51226 & 812.7319 & 878 \\
\hline Watercraft & 6.295573 & 1.565522 & 1.446547 & 3.763873 & 22.17093 & 10.22107 & 6.295573 & 1.565522 & 1.446547 & 17.94557 & 73 \\
\hline Cold & 1.740504 & 0.089662 & 0.082848 & 0.215567 & 5.261343 & 0.585387 & 1.740504 & 0.089662 & 0.082848 & 1.02779 & 11 \\
\hline Other & 2.442573 & 0.31974 & 0.295441 & 0.768729 & 7.874197 & 2.087538 & 2.442573 & 0.31974 & 0.295441 & 3.665179 & 21 \\
\hline
\end{tabular}


Undergraduate Journal of Mathematical M odeling: One + Two, Vol. 9, Iss. 2 [2019], Art. 5

\begin{tabular}{|c|c|c|c|c|c|c|c|c|c|c|c|}
\hline & $\mathrm{f} 2$ & $\mathrm{f3}$ & $\mathrm{f} 4$ & $f P$ & $\mathrm{fC}$ (female w/calf) & $\mathrm{fB}$ & $\mathrm{m} 2$ & m3 & $\mathrm{m} 4$ & $\mathrm{ma}$ & \\
\hline \multirow[t]{12}{*}{ Starting population vector: } & 77 & 72 & 71 & 184 & 194 & 500 & 77 & 72 & 71 & 878 & 2487 \\
\hline & & & & & & & & & & & Third year \\
\hline & & & & & 81.02404 & & & & & & 81 \\
\hline & 67.21546 & & & & & & & & & & 67 \\
\hline & & 69.82635 & & & & & & & & & 70 \\
\hline & & & 68.97907 & 124.6972 & & & & & & & 194 \\
\hline & & & & 54.46542 & & 149.3882 & & & & & 204 \\
\hline & & & & & 188.6747 & 337.2183 & & & & & 526 \\
\hline & & & & & 81.02404 & & & & & & 81 \\
\hline & & & & & & & 67.21546 & & & & 67 \\
\hline & & & & & & & & 69.82635 & & & 70 \\
\hline & & & & & & & & & 68.97907 & 854.4437 & 923 \\
\hline Watercraft & 5.895944 & 1.541804 & 1.523096 & 3.956009 & 23.32082 & 10.74454 & 5.895944 & 1.541804 & 1.523096 & 18.86659 & 75 \\
\hline Cold & 1.63002 & 0.088303 & 0.087232 & 0.226571 & 5.534221 & 0.615368 & 1.63002 & 0.088303 & 0.087232 & 1.080539 & 11 \\
\hline Other & 2.287524 & 0.314896 & 0.311075 & 0.80797 & 8.28259 & 2.19445 & 2.287524 & 0.314896 & 0.311075 & 3.853287 & 21 \\
\hline
\end{tabular}




\begin{tabular}{|c|c|c|c|c|c|c|c|c|c|c|c|}
\hline & $\mathrm{f} 2$ & $\mathrm{f3}$ & $\mathrm{f} 4$ & $\mathrm{fP}$ & fC (female w/calf) & $f B$ & $\mathrm{~m} 2$ & m3 & $\mathrm{m} 4$ & $\mathrm{ma}$ & \\
\hline \multirow[t]{12}{*}{ Starting population vector: } & 81 & 67 & 70 & 194 & 204 & 526 & 81 & 67 & 70 & 923 & 2590 \\
\hline & & & & & & & & & & & Forth year \\
\hline & & & & & 85.17003 & & & & & & 85 \\
\hline & 70.70158 & & & & & & & & & & 71 \\
\hline & & 65.39392 & & & & & & & & & 65 \\
\hline & & & 67.93405 & 131.1456 & & & & & & & 199 \\
\hline & & & & 57.28199 & & 157.0739 & & & & & 214 \\
\hline & & & & & 198.3292 & 354.5674 & & & & & 553 \\
\hline & & & & & 85.17003 & & & & & & 85 \\
\hline & & & & & & & 70.70158 & & & & 71 \\
\hline & & & & & & & & 65.39392 & & & 65 \\
\hline & & & & & & & & & 67.93405 & 898.398 & 966 \\
\hline Watercraft & 6.201736 & 1.443934 & 1.500021 & 4.160586 & 24.51415 & 11.29732 & 6.201736 & 1.443934 & 1.500021 & 19.83713 & 78 \\
\hline Cold & 1.714561 & 0.082698 & 0.08591 & 0.238288 & 5.817407 & 0.647027 & 1.714561 & 0.082698 & 0.08591 & 1.136124 & 12 \\
\hline Other & 2.406166 & 0.294907 & 0.306362 & 0.849753 & 8.70641 & 2.30735 & 2.406166 & 0.294907 & 0.306362 & 4.051508 & 22 \\
\hline
\end{tabular}


Undergraduate Journal of Mathematical M odeling: One + Two, Vol. 9, Iss. 2 [2019], Art. 5

\begin{tabular}{|c|c|c|c|c|c|c|c|c|c|c|c|}
\hline & $\mathrm{f} 2$ & $\mathrm{f3}$ & f4 & $\mathrm{fP}$ & fC (female w/calf) & $\mathrm{fB}$ & $\mathrm{m} 2$ & $\mathrm{~m} 3$ & $\mathrm{~m} 4$ & ma & \\
\hline \multirow[t]{12}{*}{ Starting population vector: } & 85 & 71 & 65 & 199 & 214 & 553 & 85 & 71 & 65 & 966 & 2697 \\
\hline & & & & & & & & & & & Fifth Year \\
\hline & & & & & 89.55788 & & & & & & 90 \\
\hline & 74.31937 & & & & & & & & & & 74 \\
\hline & & 68.78557 & & & & & & & & & 69 \\
\hline & & & 63.62175 & 134.8045 & & & & & & & 198 \\
\hline & & & & 58.88012 & & 165.1393 & & & & & 224 \\
\hline & & & & & 208.5468 & 372.7738 & & & & & 581 \\
\hline & & & & & 89.55788 & & & & & & 90 \\
\hline & & & & & & & 74.31937 & & & & 74 \\
\hline & & & & & & & & 68.78557 & & & 69 \\
\hline & & & & & & & & & 63.62175 & 940.1445 & 1004 \\
\hline Watercraft & 6.519078 & 1.518823 & 1.404803 & 4.276663 & 25.77708 & 11.87742 & 6.519078 & 1.518823 & 1.404803 & 20.75891 & 82 \\
\hline Cold & 1.802295 & 0.086987 & 0.080457 & 0.244936 & 6.117112 & 0.680251 & 1.802295 & 0.086987 & 0.080457 & 1.188917 & 12 \\
\hline Other & 2.529289 & 0.310202 & 0.286915 & 0.87346 & 9.154952 & 2.425828 & 2.529289 & 0.310202 & 0.286915 & 4.239772 & 23 \\
\hline
\end{tabular}


Lehigh: Population and M ortality Predictions of the Florida M anatee

\begin{tabular}{|c|c|c|c|c|c|c|c|c|c|c|c|}
\hline & f2 & f3 & $\mathrm{f} 4$ & $\mathrm{fP}$ & fC (female w/calf) & $\mathrm{fB}$ & $\mathrm{m} 2$ & $\mathrm{~m} 3$ & $\mathrm{~m} 4$ & ma & \\
\hline \multirow[t]{11}{*}{ Starting population vector: } & 90 & 74 & 69 & 198 & 224 & 581 & 90 & 74 & 69 & 1004 & 2807 \\
\hline & & & & & & & & & & & Sixth Year \\
\hline & & & & & 93.59532 & & & & & & 94 \\
\hline & 78.1482 & & & & & & & & & & 78 \\
\hline & & 72.30532 & & & & & & & & & 72 \\
\hline & & & & 58.68686 & & 173.629 & & & & & 232 \\
\hline & & & & & 217.9485 & 391.9378 & & & & & 610 \\
\hline & & & & & 93.59532 & & & & & & 94 \\
\hline & & & & & & & 78.1482 & & & & 78 \\
\hline & & & & & & & & 72.30532 & & & 72 \\
\hline & & & & & & & & & 66.92148 & 976.5642 & 1043 \\
\hline Watercraft & 6.854932 & 1.596541 & 1.477663 & 4.262626 & 26.93916 & 12.48803 & 6.854932 & 1.596541 & 1.477663 & 21.56308 & 85 \\
\hline Cold & 1.895147 & 0.091438 & 0.08463 & 0.244132 & 6.392883 & 0.715222 & 1.895147 & 0.091438 & 0.08463 & 1.234974 & 13 \\
\hline Other & 2.659595 & 0.326075 & 0.301796 & 0.870593 & 9.567675 & 2.550538 & 2.659595 & 0.326075 & 0.301796 & 4.404014 & 24 \\
\hline
\end{tabular}




\begin{tabular}{|c|c|c|c|c|c|c|c|c|c|c|c|}
\hline & $\mathrm{f} 2$ & $\mathrm{f3}$ & $\mathrm{f} 4$ & $\mathrm{fP}$ & fC (female w/calf) & $\mathrm{fB}$ & $\mathrm{m} 2$ & m3 & m4 & ma & \\
\hline \multirow[t]{12}{*}{ Starting population vector: } & 94 & 78 & 72 & 201 & 232 & 610 & 94 & 78 & 72 & 1043 & 2922 \\
\hline & & & & & & & & & & & Seventh year \\
\hline & & & & & 97.06157 & & & & & & 97 \\
\hline & 81.67127 & & & & & & & & & & 82 \\
\hline & & 76.03039 & & & & & & & & & 76 \\
\hline & & & 70.34584 & 136.2968 & & & & & & & 207 \\
\hline & & & & 59.53193 & & 182.161 & & & & & 242 \\
\hline & & & & & 226.0201 & 411.1973 & & & & & 637 \\
\hline & & & & & 97.06157 & & & & & & 97 \\
\hline & & & & & & & 81.67127 & & & & 82 \\
\hline & & & & & & & & 76.03039 & & & 76 \\
\hline & & & & & & & & & 70.34584 & 1015.207 & 1086 \\
\hline Watercraft & 7.163965 & 1.678793 & 1.553275 & 4.324006 & 27.93684 & 13.10168 & 7.163965 & 1.678793 & 1.553275 & 22.41634 & 89 \\
\hline Cold & 1.980584 & 0.096149 & 0.08896 & 0.247647 & 6.62964 & 0.750367 & 1.980584 & 0.096149 & 0.08896 & 1.283842 & 13 \\
\hline Other & 2.779495 & 0.342874 & 0.317239 & 0.883129 & 9.922008 & 2.67587 & 2.779495 & 0.342874 & 0.317239 & 4.578283 & 25 \\
\hline
\end{tabular}




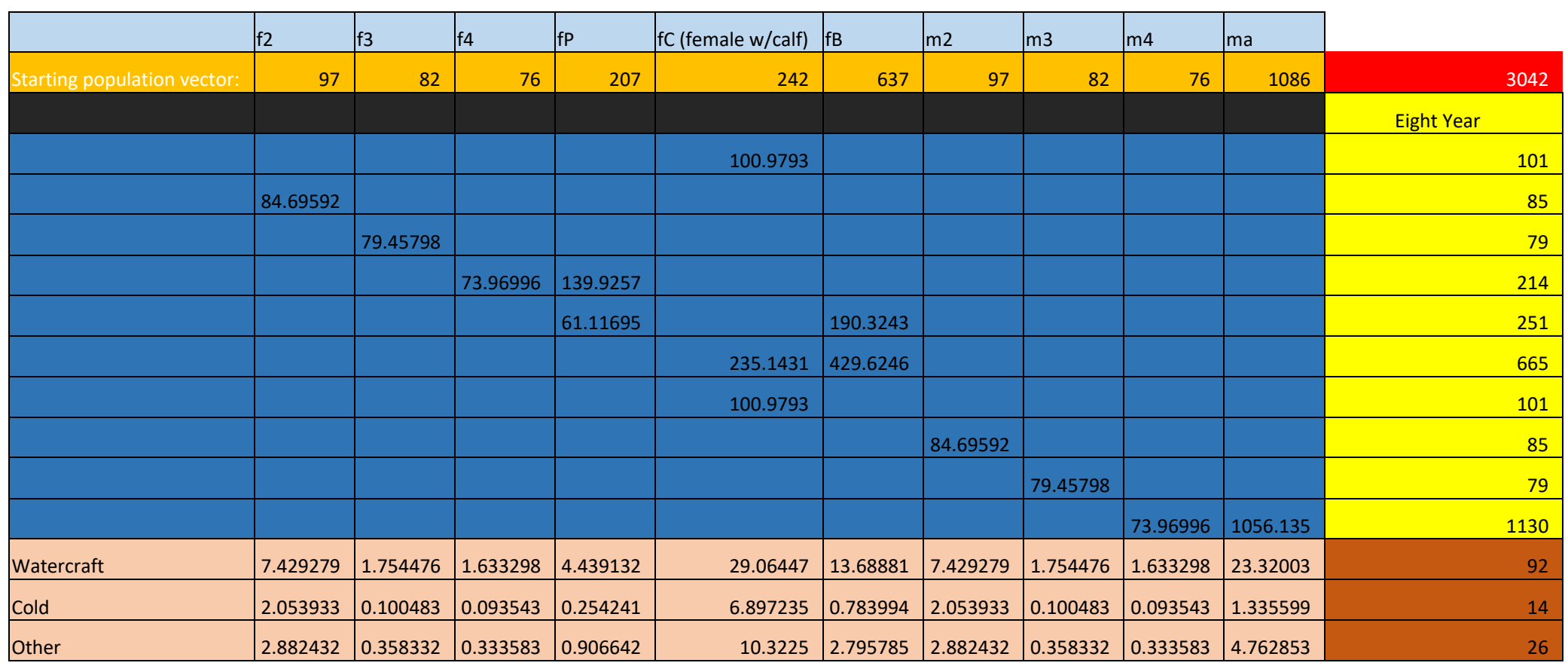




\begin{tabular}{|c|c|c|c|c|c|c|c|c|c|c|c|}
\hline & $\mathrm{f} 2$ & f3 & $\mathrm{f4}$ & $\mathrm{fP}$ & fC (female w/calf) & $f B$ & $\mathrm{~m} 2$ & m3 & $\mathrm{m} 4$ & $\mathrm{ma}$ & \\
\hline \multirow{11}{*}{ Starting population vector: } & 101 & 85 & 79 & 214 & 251 & 665 & 101 & 85 & 79 & 1130 & 3167 \\
\hline & & & & & & & & & & & Ninth Year \\
\hline & & & & & 105.0522 & & & & & & 105 \\
\hline & 88.11455 & & & & & & & & & & 88 \\
\hline & & 82.40066 & & & & & & & & & 82 \\
\hline & & & 77.30467 & 144.8369 & & & & & & & 222 \\
\hline & & & & 63.26211 & & 198.553 & & & & & 262 \\
\hline & & & & & 244.6272 & 448.1994 & & & & & 693 \\
\hline & & & & & 105.0522 & & & & & & 105 \\
\hline & & & & & & & 88.11455 & & & & 88 \\
\hline & & & & & & & & 82.40066 & & & 82 \\
\hline Watercraft & 7.729151 & 1.819452 & 1.70693 & 4.594942 & 30.23674 & 14.28065 & 7.729151 & 1.819452 & 1.70693 & 24.2771 & 96 \\
\hline Cold & 2.136837 & 0.104205 & 0.09776 & 0.263164 & 7.175424 & 0.81789 & 2.136837 & 0.104205 & 0.09776 & 1.390413 & 14 \\
\hline Other & 2.998777 & 0.371603 & 0.348621 & 0.938465 & 10.73884 & 2.916661 & 2.998777 & 0.371603 & 0.348621 & 4.958322 & 27 \\
\hline
\end{tabular}




\begin{tabular}{|c|c|c|c|c|c|c|c|c|c|c|c|}
\hline & f2 & $\mathrm{f3}$ & $\mathrm{f4}$ & $\mathrm{fP}$ & $\mathrm{fC}$ (female w/calf) & $\mathrm{fB}$ & $\mathrm{m} 2$ & m3 & $\mathrm{m} 4$ & ma & \\
\hline \multirow[t]{11}{*}{ Starting population vector: } & 105 & 88 & 82 & 222 & 262 & 693 & 105 & 88 & 82 & 1177 & 3296 \\
\hline & & & & & & & & & & & Tenth Year \\
\hline & & & & & 109.3864 & & & & & & 109 \\
\hline & 91.66851 & & & & & & & & & & 92 \\
\hline & & 85.72664 & & & & & & & & & 86 \\
\hline & & & & 65.70096 & & 206.9337 & & & & & 273 \\
\hline & & & & & 254.7199 & 467.1174 & & & & & 722 \\
\hline & & & & & 109.3864 & & & & & & 109 \\
\hline & & & & & & & 91.66851 & & & & 92 \\
\hline & & & & & & & & 85.72664 & & & 86 \\
\hline & & & & & & & & & 80.16761 & 1144.893 & 1225 \\
\hline Watercraft & 8.040894 & 1.892892 & 1.770145 & 4.772084 & 31.48423 & 14.88342 & 8.040894 & 1.892892 & 1.770145 & 25.27986 & 100 \\
\hline Cold & 2.223023 & 0.108411 & 0.101381 & 0.27331 & 7.471465 & 0.852412 & 2.223023 & 0.108411 & 0.101381 & 1.447844 & 15 \\
\hline Other & 3.119728 & 0.386602 & 0.361532 & 0.974644 & 11.1819 & 3.03977 & 3.119728 & 0.386602 & 0.361532 & 5.163125 & 28 \\
\hline
\end{tabular}

